\title{
Análisis de los ciclos del producto interno bruto agropecuario colombiano 1976-2013*
}

\author{
Analysis of the Cycles of the Colombian \\ Agricultural GDP 1976-2013
}

\section{Análise do ciclos do PIB agrícola colombiano 1976-2013}

Álvaro Hernando Chaves Castro**

Este trabajo forma parte de una investigación más completa sobre un diagnóstico del sector agropecuario que se desarrolló con recursos de la Escuela Colombiana de Ingeniería. El autor agradece al estudiante Juan Camilo Osorio del Programa de Economía por la excelente labor como asistente de investigación. De igual manera agradece al doctor Enrique López Enciso del Banco de la República por sus valiosos comentarios a una versión anterior del artículo.

** Economista y magíster en Ciencias Económicas. Profesor asociado e investigador del Centro de Estudios Económicos del Programa de Economía en la Escuela Colombiana de Ingeniería, Bogotá, Colombia. alvaro. chaves@escuelaing.edu.co 


\section{Resumen}

El análisis de contabilidad de crecimiento en el sector entre el periodo 1976-2013 muestra una fuerte contribución del factor trabajo y del crecimiento de la PTF, pero una baja contribución del stock de capital. Los resultados de la estimación del ciclo a partir de diferentes métodos de filtrado de series de tiempo indican que se registran tres ciclos completos con una duración de 16 trimestres (4 años). Las fases de expansión duran, en promedio, 29 trimestres (9,6 años), y las de contracción, 4 trimestres (1 año), lo que implica que los ciclos en el sector agrícola son asimétricos, dada la mayor duración de las expansiones con relación a las contracciones. El análisis de comovimientos del ciclo del PIB y cada uno de sus componentes de demanda en el sector muestra que las variables agregadas de la actividad agropecuaria se comportan de manera procíclica. La fuerte covarianza del PIB con el consumo aparente y de las exportaciones del café y la balanza comercial es uno de los mejores hechos estilizados que han caracterizado las fluctuaciones económicas en el sector agrícola durante el periodo de análisis.

Palabras clave: ciclo, productividad multifactorial, hecho estilizado, comovimiento, Producto Interno Bruto.

Códigos JEL: E32, C32, Q1, D24

\section{Abstract}

The analysis of growth accounting in agricultural sector during 1976-2013 shows a strong contribution of labor factor and TFP, but a low contribution of capital. The results of the cycle estimation using different methods of time series filtering indicate the existence of three complete cycles with duration of 16 quarters (4 years). The expansions remain for 29 quarters on average ( 9.6 years) and contractions 4 quarters (1 year), which implies that cycles in agricultural sector are asymmetric given the long duration of expansions compared to contractions. The co-movement analysis of the agricultural GDP cycle and each of its demand components shows that aggregate variables of agricultural activity behave procyclically. The strong covariance of GDP with apparent consumption and of coffee exports and trade balance is one of the best stylized facts that have characterized the economic fluctuations in the Colombian agricultural sector during this period.

Keywords: cycle, total factor productivity, stylized fact, co-movement, GDP. 


\section{Resumo}

A análise da contabilidade do crescimento do setor entre o período 1976-2013, mostra um forte contributo do trabalho fator, baixa contribuição do estoque de capital e forte contribuição do crescimento da TFP. Os resultados da estimativa do ciclo de diferentes métodos de filtragem de séries temporais, indicam que registar-se três ciclos completos, com uma duração de 16 trimestres (4 anos). Períodos de recuperação duran, em média, 29 trimestres (9,6 anos) e contração dos 4 trimestres ( 1 ano), implicando que os ciclos no sector agrícola são assimétricos dado mais expansões em comparação com as contrações. Análise do dos co - movimentos do ciclo da PIB e cada um dos componentes da demanda no setor, mostram que as variáveis adicionadas de atividade agrícola é se comportar de modo cíclico. A covariância forte do PIB com consumo aparente e as exportações de café e a balança comercial, é um dos melhores fatos estilizados que tem caracterizado as flutuações económicas no sector agrícola durante o período de análise.

Palavras-chave: ciclo, produtividade multifator, fez estilizado. 


\section{INTRODUCCIÓN}

La relación entre el ciclo y el crecimiento económico ha sido muy estudiada en economía y ha sido objeto de grandes debates. En el trabajo de Zarnowitz (1992) se analiza detalladamente la relación de estos dos fenómenos a partir de la siguiente pregunta: ¿Se encuentra la tendencia a largo plazo (crecimiento) de la economía fuertemente influenciada por las fluctuaciones a corto plazo (ciclos), y si es así, cómo? De acuerdo con el autor, esta es una de las mayores inquietudes que ha recibido una considerable atención en la literatura económica. Sin embargo, se ha aceptado generalmente que el proceso de crecimiento a largo plazo es "real" por naturaleza, explicado por los aumentos en la cantidad de recursos físicos y por la productividad del capital humano.

Por otro lado, la teoría del ciclo de los negocios discrepa de la teoría del crecimiento en cuanto al papel que juegan los factores reales y monetarios, es decir, en el papel de las políticas de demanda agregada para alterar el ciclo de la economía; sin embargo, en principio, no existen objeciones sobre la idea de que la tendencia y el ciclo son separables. En la práctica, esta concepción permite la estimación y el análisis del "crecimiento de los ciclos económicos", en cuanto los movimientos en el nivel de actividad agregada están definidos por el comportamiento de diferentes indicadores agregados, ajustados por su tendencia a largo plazo. No obstante, esta visión del ciclo, manifestada como las fluctuaciones observadas de la producción con respecto a la tendencia a largo plazo, ha estado sujeta a fuertes críticas y dificultades en la estimación.

Al analizar la dinámica del producto interno bruto agropecuario colombiano a lo largo del tiempo, se debe hacer una estimación de los ciclos, con el fin de identificar las diferentes fases, la 
amplitud y la duración de estos. Dicho ejercicio puede realizarse a partir de un enfoque microeconómico, que identifique y estime los ciclos particulares de cada producto, dada su naturaleza transitoria o permanente, o desde un enfoque agregado, que analice las series de tiempo del PIB del sector y aplique un conjunto de métodos de filtrado para estimar las fases de la actividad del sector.

El presente trabajo parte de una descripción agregada de la dinámica de la actividad agropecuaria y su relación con el comportamiento agregado de la economía, mediante la estimación y el análisis de los ciclos del PIB agrícola y su relación con variables de oferta y demanda.

El enfoque utilizado en este trabajo, en la estimación del ciclo con base en una gran batería de datos de series de tiempo, permite un análisis diferente y se centra en la descripción de la naturaleza del ciclo del PIB agropecuario; además es relevante, porque da indicios y ofrece elementos macroeconómicos para resaltar algunos cambios estructurales experimentados por el sector en este lapso de tiempo.

Algunos estudios en Colombia han analizado ciertas hipótesis en torno a los ciclos y cambios estructurales del sector agropecuario, como los de Londoño (1984), Kalmanovitz y López (2006), Jaramillo (2002), entre otros, los cuales se caracterizan por no soportar los resultados en hallazgos empíricos fuertes y no resaltar los impactos de las políticas implementadas en el sector sobre cada una de las fases del ciclo.

El trabajo parte de la hipótesis según la cual los ciclos del PIB agropecuario son irregulares, en el sentido de que las fases presentan duraciones distintas, comparados con el PIB total en Colombia, y que variables por el lado de la demanda, como los flujos de comercio internacional, se comportan de manera procíclica con el ciclo estimado del PIB agropecuario.

El presente trabajo está estructurado en seis secciones. La primera es esta introducción. En la segunda parte se describen los diferentes hechos estilizados relacionados con el PIB del sector agrícola. En la sección tercera se estiman e interpretan los ciclos del sector, destacando aspectos relacionados con la duración, la frecuencia y su asimetría, a partir de diferentes métodos de filtrado. En esta misma sección se expone la metodología de métodos de filtrado, pues esta es la que permitirá la estimación del ciclo; además es de gran relevancia porque implica analizar las propiedades estadísticas de las series de tiempo involucradas en el análisis. En la sección cuarta se estudia el comportamiento estocástico del PIB agropecuario, cuya finalidad es estimar la duración de los choques exógenos sobre el ciclo del PIB. En la quinta parte se analizan los comovimientos del ciclo agropecuario con variables de oferta y demanda, evaluando las correlaciones rezagadas y contemporáneas, y se destaca el papel de las políticas contracíclicas 
implementadas por el gobierno durante el periodo de análisis. Y en último término se presentan unos comentarios a manera de conclusión.

\section{HECHOS ESTILIZADOS EN LA ACTIVIDAD AGROPECUARIA}

Uno de los fenómenos económicos en los que existe consenso por parte de los economistas es el relacionado con las fluctuaciones o movimientos del producto, el empleo y los precios. La teoría del crecimiento económico ha explicado los factores que determinan el crecimiento a largo plazo de las economías, las expansiones y recesiones que se presentan a lo largo del tiempo que están asociadas con movimientos en el nivel de empleo. Las fases recesivas de la actividad económica normalmente están acompañadas de largos periodos de alto desempleo y en algunos casos por periodos de deflación, en donde el nivel general de precios, entre otras cosas, tiende a caer debido a una débil demanda agregada.

El nivel de actividad agrícola colombiano ha estado sujeto a grandes fluctuaciones $\mathrm{y}$ al mismo tiempo ha jugado un papel central en el desempeño global de la economía del país. En efecto, desde una perspectiva a largo plazo, Colombia adoptó una estrategia de crecimiento de la actividad agrícola, caracterizada por una rápida expansión de la producción de café y de las exportaciones durante finales del siglo 19 y comienzo del siglo 20 (Berry, 1970). De acuerdo con Ocampo $(1984,1991)$, a lo largo del siglo 20 la participación del PIB agrícola con relación al PIB total ha estado influenciada por la dinámica de la producción de café y ha sido el principal producto exportable por varios años en este periodo.

\section{Dinámica y comportamiento del PIB agropecuario}

Uno de los hechos macroeconómicos sobresalientes durante los últimos 37 años en Colombia ha sido el crecimiento del PIB del sector agrícola. La Figura 1 describe el comportamiento del PIB real agropecuario de la economía colombiana durante el periodo 1975 a 2013. La tasa de crecimiento promedio durante los últimos 37 años fue de $2.6 \%$. 


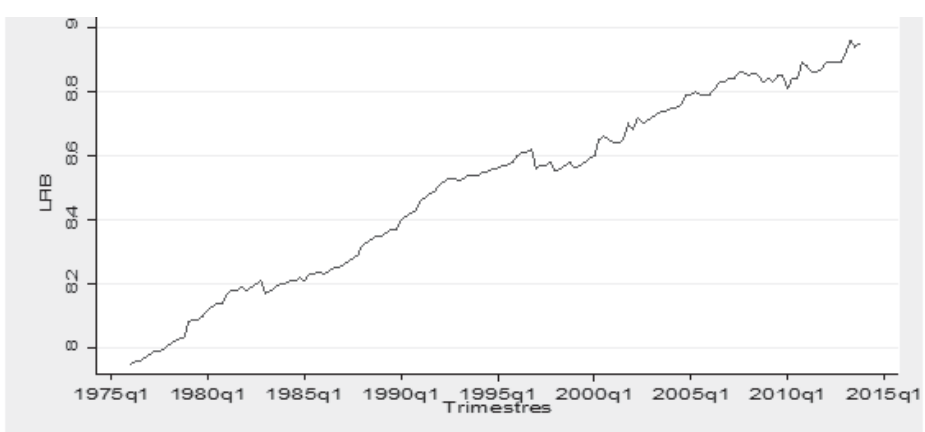

Figura 1. Logaritmo del PIB agropecuario: 1976-I a 2013-IV.

Fuente: cálculos propios sobre cifras DANE, Sistemas de Cuentas Nacionales. Se hizo un empalme mediante retropolación con aplicación de tasas de crecimiento.

No obstante, el nivel de actividad del sector ha presentado periodos de auge y caídas, tanto en su nivel como en la tasa de crecimiento. En efecto, en la segunda mitad de la década de los 70, el PIB agropecuario creció a una tasa promedio de $3.9 \%$, entre 1981 y 1997 creció $2.5 \%$, y entre 1998 y 2013 el crecimiento promedio del sector fue de $2.4 \%$. Lo anterior implica que el PIB del sector en la actualidad es cerca de 2.3 veces mayor que el de finales de los 70 , 1.4 veces mayor que el del 90 , y 1.3 veces más grande que en el año 2000.

La tasa de crecimiento promedio más alta se alcanzó a finales de la década de los 70, periodo que coincide con el boom exportador del sector cafetero y que de acuerdo con autores como Berry (1970) y Ocampo (1984), la importancia del PIB agropecuario en el total de la economía se alcanzó una vez que el café se convirtió en el sector líder del país. No obstante, en los 80 y finales de los 90 la dinámica del PIB del sector se vio afectada negativamente por fenómenos como la caída de precios internacionales en los 80 y la recesión del año 99, que afectó negativamente a la mayoría de sectores económicos.

\section{Fuentes de crecimiento del sector agropecuario}

Existe una serie de hechos estilizados del crecimiento económico que podrían explicar desde el punto de vista macroeconómico la dinámica del PIB agropecuario. El primero de ellos es que el crecimiento del producto refleja el aumento tanto de la mano de obra, la tierra, la maquinaria, así como de la productividad laboral. Para el caso del sector agropecuario, el crecimiento promedio del empleo entre 1976 y 2013 fue de $2.4 \%$, muy cercano al crecimiento promedio obtenido por el PIB agropecuario en este mismo periodo; mientras que la productividad laboral del sector aumentó a una tasa promedio de $0.09 \%$, menos de un punto porcentual. La dinámica del PIB agropecuario en este periodo ha evolucionado a la par con el crecimiento del factor trabajo, tal como se evidencia en la Figura 2. 


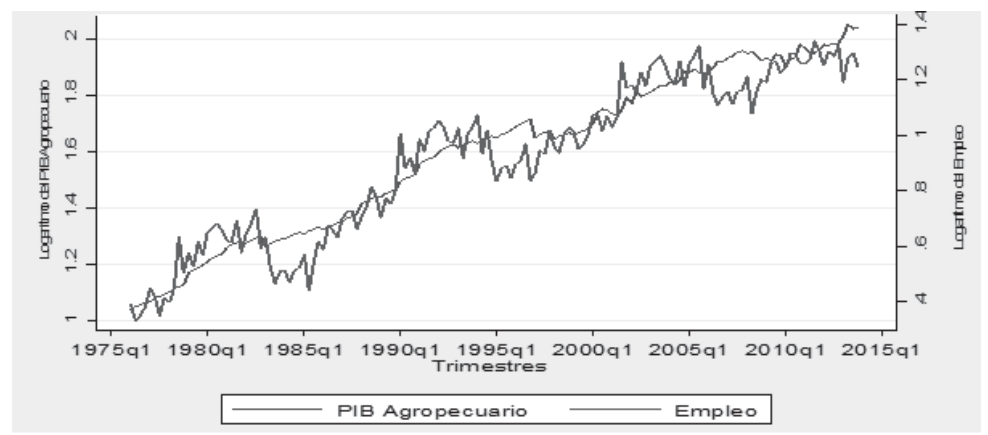

Figura 2. Comportamiento del PIB y el empleo en el sector agropecuario 1976-I a 2013-IV. Fuente: cálculos propios sobre cifras DANE: Sistema de Cuentas Nacionales y Gran Encuesta Integrada de Hogares.

La Figura 2 revela la dinámica del PIB agropecuario y el nivel de empleo durante los últimos 37 años, en la escala derecha se mide el logaritmo del empleo y en la escala izquierda el logaritmo del PIB. En general, el empleo y el producto durante este periodo exhiben una tendencia creciente, con la característica de importantes fluctuaciones del empleo alrededor de la tendencia del PIB. Parte de las fuertes fluctuaciones del empleo en el sector agrícola se debe a los factores de tipo estacional presentes en cada una de las actividades del sector y también a algunos factores demográficos producto de flujos migratorios de la mano de obra entre actividades. De acuerdo con Londoño (1984), en una economía con bajos niveles de ingreso, el sector agropecuario constituye la fuente primordial de empleo de la población; y uno de los hechos estilizados del desarrollo es la disminución relativa de esta importancia del sector rural como generador de empleo y el desplazamiento de la mano de obra hacia otros sectores.

En cuanto al comportamiento del empleo en el sector existen unos episodios bien marcados. Por ejemplo, la tasa de crecimiento promedio entre 1980 y 1976 fue de $2.9 \%$, entre 1990 y 1980 fue de $1.8 \%$, y entre 2013 y 2000 fue de apenas $1.2 \%$. Las anteriores tasas de crecimiento del factor empleo podrían reflejar una sustitución entre factores productivos en el interior del sector y un aumento en la productividad de las actividades agropecuarias ${ }^{1}$. En efecto, el nivel de mano de obra disponible en el sector en 1980 fue 1.5 veces más grande del existente a mediados de la década

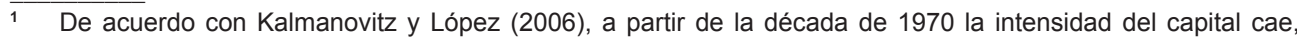
acompañada de una creciente productividad de todos los factores, que se acelera en especial en la última década del siglo XX. No obstante, de acuerdo con los autores, el uso del factor trabajo se mantuvo constante a lo largo de los primeros cuarenta años del medio siglo y se intensifica en la última década. 
de los 70, el de 1990 fue 1.4 veces más grande que el de 1980 y el del 2013 fue 1.3 veces más alto que el de comienzos de esta década.

Dicha trayectoria agregada del PIB y el empleo en este periodo se encuentra cercana al hecho estudiado en trabajos seminales sobre ciclos económicos como los de Okun (1962), Prescott (1986) y Campbell y Mankiw (1987a), entre otros, quienes defienden la idea de que el nivel de actividad de la economía fluctúa alrededor de un tendencia suave a largo plazo de la producción.

Por el momento, la principal conclusión que se puede extraer de este primer hecho estilizado es el comportamiento tendencial entre el nivel de actividad económica y el empleo en el sector agropecuario. Dicho comportamiento del empleo, al parecer, no es novedoso, pues en el trabajo de Londoño (1984) se observa que a partir del decenio la reasignación de la mano de obra en Colombia ha resultado mucho más veloz de lo que cabría esperarse en la experiencia de otros países. El autor, a partir de un análisis estructural a largo plazo, concluye que la rápida movilidad espacial de la fuerza de trabajo desde comienzos de la década del cincuenta contribuyó a que a partir de este periodo de quiebre, la brecha de productividades en el sector rural se acortara más rápido de lo que los patrones internacionales permitieran esperar.

El segundo hecho estilizado en el aspecto macroeconómico en el comportamiento del PIB tiene que ver con la contribución de los factores productivos como capital, empleo y conocimiento (estado de la tecnología) sobre el crecimiento de la producción del sector. Con el fin de corroborar dicha hipótesis, se analiza la contabilidad de crecimiento de la producción agropecuaria, que consiste en estimar el efecto de factores productivos como el empleo, stock de capital y del crecimiento de la productividad total de los factores (PTF), que está en consonancia con los trabajos originales de Solow (1957) y de otros más recientes como los de Barro y Sala-i-Martin (2004).

La estimación de la PTF para el sector agrícola introduce el efecto del aumento del capital inducido por el incremento de la eficiencia productiva. De esta forma, se estima la contribución de los factores productivos capital y mano de obra en el crecimiento del PIB agropecuario, introduciendo este efecto tal como se hace en el trabajo de Hayashi y Prescott (2008), quienes parten de la siguiente definición de la productividad total de los factores (TFP):

$$
T F P \equiv Y_{t} / K_{t}^{\theta}\left(h_{t} E_{t}\right)^{1-\theta}
$$

Donde $y_{t}$ es el PIB del sector agrícola en el periodo t, $K_{t}$ es el stock de capital, $E_{t}$ es el nivel de empleo, $h_{t}$ es el número de horas promedio trabajadas por persona, $\left(h_{t} E_{t}\right)$, igual al total de horas trabajadas, y $\theta$ es la participación de los ingresos del capital en el total del producto. A partir de las estimaciones econométricas previas, se encontró un valor del parámetro 
$\theta=0.55$, que es el que se utilizará para el análisis de contabilidad de crecimiento. Al emplear un poco de álgebra en la definición de la PTF, se puede encontrar una expresión para el PIB por trabajador, que se puede descomponer en cuatro factores, de la siguiente manera:

$Y_{t} / N_{t}=T F P_{t}^{1 / 1-\theta} *\left(\mathrm{~K}_{t} / Y_{t}\right)^{\theta / 1-\theta} *\left(E_{t} / N_{t}\right) * h_{t}$

Donde $N_{t}$ es la población en edad de trabajar. La expresión [2] muestra que a largo plazo, donde la relación capitalproducto $\left(K_{t} / Y_{t}\right)$, la tasa de ocupación $\left(E_{t} / N_{t}\right)$ y las horas trabajadas por persona son constantes, la tendencia del producto por trabajador $\left(Y_{t} / N_{t}\right)$ está dada por la PTF, es decir por el factor $T F P_{t}{ }^{1 / l-\theta}$. Según Hayashi y Prescott (2008), la potencia 1/ $(1-\theta)$ refleja el hecho de que un aumento de la productividad total de los factores (TFP) genera un incremento proporcional en el stock de capital, por tanto el factor capital-producto $\left(K_{t} / Y_{t}\right)^{\theta /(1-\theta)}$, representa únicamente la parte de la acumulación del capital no inducida por el crecimiento de la $\mathrm{TFP}^{2}$. El lado izquierdo de la ecuación [2] se presenta en la Figura 3. Es importante resaltar que en la estimación econométrica del parámetro $\theta$, adicional al método de estimación por mínimos cuadrados ordinarios (MCO), se utilizó una estimación recursiva de MCO que permite que las participaciones factoriales sean variables en el tiempo ${ }^{3}$.

La Tabla 1 presenta la tasa de crecimiento promedio anual del PIB por trabajador y sus cuatro factores que sugiere la expresión [4] durante el periodo 1976 a 2012.

$2 \quad$ Esta fórmula ha sido adoptada por King y Levine (1994), Hayashi y Prescott (2002), y otros. Klenow y RodríguezClare (1997) discuten las ventajas y desventajas de esta forma para abordar la contabilidad del crecimiento comparada con la forma estándar de descomposición del producto entre la TFP, crecimiento del capital y de la mano de obra.

3 Sin embargo, los resultados de MCO recursivos en magnitud son muy cercanos a los encontrados por MCO con parámetros fijos o constantes en el tiempo. Esto implica que a pesar de que estos varían en el tiempo, las participaciones de los diferentes factores en el producto son relativamente estables durante el periodo de análisis. 


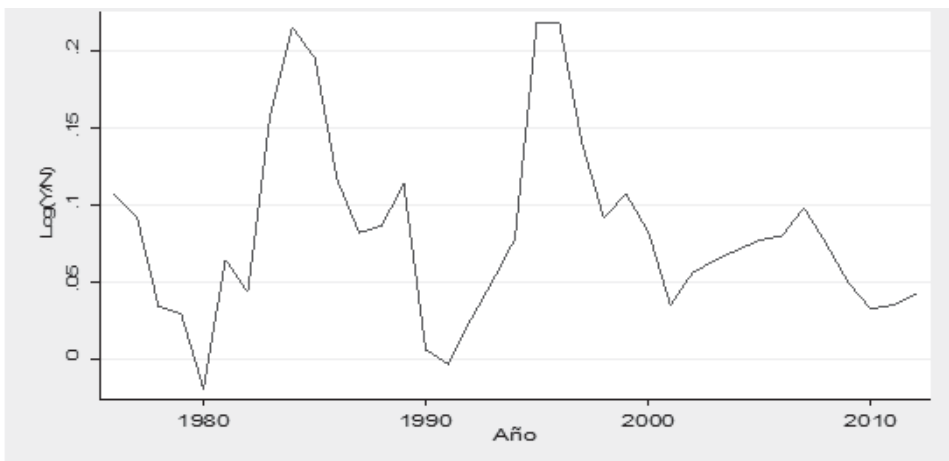

Figura 3. PIB por trabajador en sector agrícola 1976-2012.

Fuente: cálculos propios sobre cifras DANE: Sistema de Cuentas Nacionales.

La variable PIB se encuentra en pesos constantes del año 2005.

Para el periodo 1976 a 1980 se presenta una caída en la tasa de crecimiento promedio del producto por trabajador de $-1.16 \%$, que es consistente con la disminución experimentada por la TFP, a pesar de que la relación capital-producto se incrementó significativamente $(1.42 \%)^{4}$. Para la década comprendida entre los años 1980 a 1990, el producto por trabajador se recupera y experimenta un menor decrecimiento promedio de $0.97 \%$, un poco por debajo del crecimiento experimentado por la TFP que fue de $0.58 \%$.

El crecimiento de la productividad del sector después de la década de los noventa es notable y compensó el pobre desempeño de la relación capital-producto y del nivel de empleo en el sector. En efecto, la tasa de crecimiento promedio de la TFP fue de $2.24 \%$ entre 1990 y 2000 y de $2.52 \%$ entre 2000 y 2012. El crecimiento del producto por trabajador en este periodo creció en promedio $1,29 \%$ entre 1990 y 2000, y decreció $-0.36 \%$ en el periodo 2000 a 2012. El factor que describe la relación capital-producto después de experimentar un crecimiento vigoroso de $1.42 \%$ en la segunda mitad de los 70 , ha presentado un decrecimiento sostenido en las posteriores décadas, y sugiere que su dinámica a lo largo del tiempo no ha sido inducida por el crecimiento del factor de la TFP, dada la especificación adoptada para la función de producción (Figura 3).

\footnotetext{
$4 \quad$ Dado que la agricultura es típicamente una actividad atomizada, con muy pocos incentivos para que los agricultores implementen actividades de investigación en sus procesos productivos, las tasas de crecimiento en la productividad total de los factores reflejan ganancias en eficiencias y un fenómeno de reasignación de recursos en el sector agropecuario colombiano.
} 
Tabla 1. Fases del ciclo del PIB del sector agropecuario

\begin{tabular}{|c|c|c|c|c|c|}
\hline Periodo & $\begin{array}{c}\text { PIB por } \\
\text { trabajador } \\
\mathbf{Y}_{\mathbf{t}} / \mathbf{N}_{\mathbf{t}}\end{array}$ & $\begin{array}{c}\text { Factor } \\
\mathbf{T F P} \\
\mathbf{T F P}\end{array}$ & $\begin{array}{c}\text { Relación capi- } \\
\text { tal-producto } \\
\left(\mathbf{K}_{\mathbf{t}} / \mathbf{Y}_{\mathbf{t}} \mathbf{)}\right.\end{array}$ & $\begin{array}{c}\mathbf{\theta / ( 1 - \boldsymbol { \theta } )} \\
\text { Tasa de } \\
\text { ocupación } \\
\mathbf{E}_{\mathbf{t}} / \mathbf{N}_{\mathbf{t}}\end{array}$ & $\begin{array}{c}\text { Horas trabajadas } \\
\text { por trabajador } \\
\mathbf{h}_{\mathbf{t}}\end{array}$ \\
\hline $1976-1980$ & $-1,16$ & $-3,85$ & 1,42 & 1,37 & $-0,09$ \\
$1980-1990$ & $-0,97$ & 0,58 & $-0,34$ & $-1,20$ & 0,00 \\
$1990-2000$ & 1,29 & 2,24 & $-1,41$ & 0,49 & $-0,03$ \\
$2000-2012$ & $-0,36$ & 2,52 & $-2,29$ & $-0,53$ & $-0,07$ \\
\hline
\end{tabular}

Fuente: cálculos propios sobre cifras DANE, Sistema de Cuentas Nacionales.

Nota: promedio geométrico. $\mathrm{Y}_{1}$ : PIB real; $\mathrm{K}_{1}$ : stock de capital; $\mathrm{E}_{1}$ : empleo; $\mathrm{N}_{1}$ : población en edad de trabajar; $\mathrm{h}_{\mathrm{t}}$ : horas promedio por persona empleada.

Los análisis de estimación de la PTF y contabilidad del crecimiento del PIB agropecuario realizados en esta sección permiten extraer las siguientes conclusiones. Uno de los hechos macroeconómicos más importantes durante el periodo 1976 y 2013 fue el crecimiento persistente del PIB por trabajador del sector agropecuario, sobre todo en el periodo de 1980 a 2000. El crecimiento del PIB agropecuario durante este periodo se debe en gran parte al crecimiento de la PTF, más que a la expansión en el producto por trabajador, similar a lo encontrado por autores como Kalmanovitz y López (2006).

La contribución del cambio tecnológico al crecimiento del PIB del sector ha presentado una tendencia creciente a lo largo de estos 37 años. Por su parte, la relación capital-producto ha mostrado una tendencia decreciente, excepto en el periodo 1976 a 1980, cuando la participación del stock de capital del sector con relación al PIB se favoreció en parte por los recursos derivados de la bonanza cafetera que permitió la importación de tractores y maquinaria agrícola, y en parte por el aumento en la tasa de ahorro.

El análisis de contabilidad de crecimiento durante este periodo muestra una importante contribución del factor trabajo y una fuerte contribución del crecimiento de la PTF. Este hecho coincide con el análisis de autores como Kalmanovitz y López (2006) y el Grupo de Estudios del Crecimiento Económico GRECO (2004), según el cual, las formas modernas de producir en el sector se hicieron dominantes y el desenvolvimiento de la economía colombiana se fue haciendo cada vez más dependiente de la acumulación de los factores productivos y del aumento de la población económicamente activa en el sector; se estimuló la configuración de una modalidad de crecimiento basada en 
el uso intensivo de factores (Kalmanovitz \& López, 2006, p. 16).

En la Figura 4 se presenta la evolución de la relación capital-producto durante el periodo de análisis. Después de alcanzar un máximo en el año 1983, en que la relación experimentó un crecimiento de $14 \%$ con relación al año 1976 , se observa una tendencia decreciente hasta alcanzar un mínimo en el año 2012, cuando la relación experimentó un crecimiento negativo de $-35.6 \%$. La disminución sostenida desde 1983 de la relación capital-producto podría estar explicada por menores inversiones en el sector agrícola, que coincide con un descenso vertiginoso en el inventario de tractores que comienza a partir del año 1983 (Uribe, 1998), y por otra parte, de un incremento del producto por trabajador al utilizar intensivamente la mano de obra ${ }^{5}$.

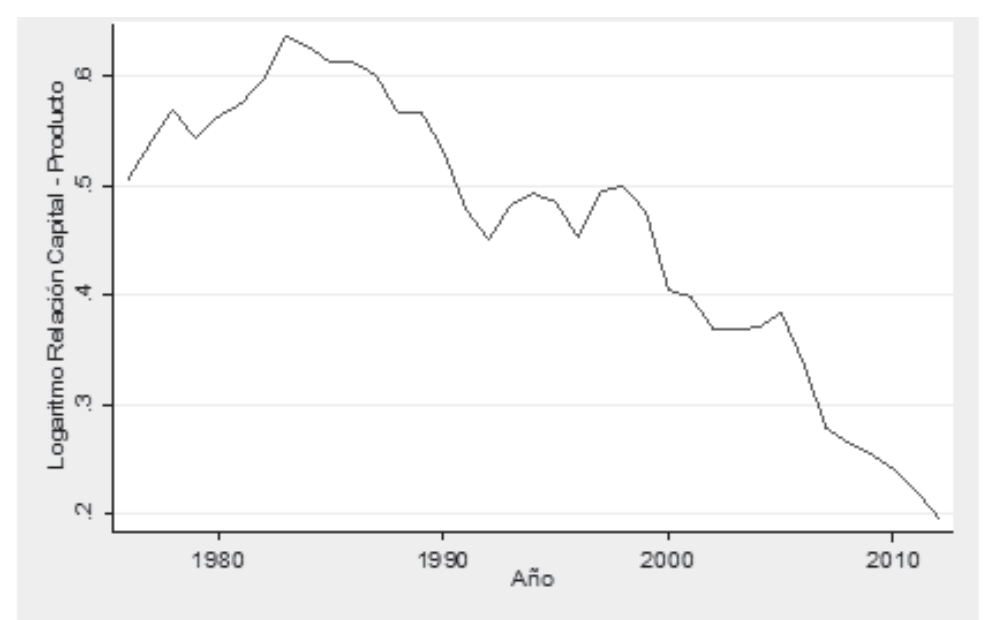

Figura 4. Relación capital-producto en el sector agropecuario 1976-2012.

Fuente: cálculos propios sobre cifras DANE: Sistema de Cuentas Nacionales. Las variables PIB y stock de capital se encuentran en pesos constantes del año 2005.

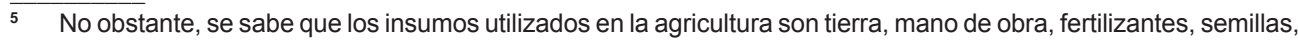
maquinaria y riego. Adicionalmente, su importancia varía de acuerdo con las características del cultivo, la zona de producción y el país. 
Sin embargo, entre 1991 y 1995 la relación capital-producto experimentó una tasa de crecimiento positivo de $5.3 \%$, explicado en parte por el aumento en las importaciones de maquinaria agrícola que estimuló la rebaja arancelaria en el marco de la apertura económica en este periodo.

En efecto, la importación promedio anual de tractores en los años noventa fue de 585 unidades, y el número de tractores entre 1991 y 1995 pasó de 523 tractores a 811 tractores, lo que equivale a una tasa de crecimiento de $55 \%$.

De acuerdo con Urrutia y Robinson (2007), parte de la financiación de la inversión agregada de la economía se debió al ahorro externo y se dio con mayor intensidad entre 1993 y 1995, hecho que podría ayudar a explicar la recuperación de la relación capitalproducto entre 1991 y 1995.

En el trabajo del GRECO del Banco de la República se hace una excelente revisión de los trabajos que han ofrecido evidencia empírica en torno a la elasticidad de la demanda por importaciones de bienes de capital. De acuerdo con dicha revisión se encuentra que la elasticidad de la demanda de importaciones de bienes de capital con respecto a los precios, son bastante altas para el sector agrícola y además estas son bastante sensibles a las restricciones cuantitativas al comercio internacional. Por consiguiente, es posible que en la apertura de la década de los noventa, la demanda por importaciones de bienes de capital aumentara en el sector agropecuario con relación a la importación de otro tipo de bienes.

\section{MEDICIÓN E INTERPRETACIÓN DEL CICLO DEL PIB AGROPE- CUARIO Y LAS POLÍTICAS ECO- NÓMICAS}

El PIB agropecuario, al igual que el PIB total de la economía, ha estado sujeto a periodos de auge y caídas en la actividad económica, que podrían estar explicados por factores tanto de oferta como de demanda. Esta sección ofrece, en primer lugar, una estimación del ciclo en términos macroeconómicos para el PIB agropecuario y una interpretación de las diferentes fases comprendidas en los últimos 37 años que abarca el periodo 1976 a $2013^{6}$.

\section{Estimación de la tendencia y el ciclo del PIB agropecuario}

De acuerdo con Blanchard y Fischer (1986), el primer estudio sistemático del ciclo a partir de un enfoque de series de tiempo fue realizado por Burns and Mitchell (1946), cuya metodología se plasmó en el libro Measuring Business Cycles, publicado por el National Bureau Economic Research -NBER- en el año

\footnotetext{
6 En estricto sentido, una estimación más completa del ciclo en el sector agropecuario requiere tener en cuenta los componentes estacionales en cada una de las actividades del sector, tanto agrícolas (cultivos) como pecuarias. No obstante, la metodología que se aborda en el presente trabajo es desde el punto de vista de la econometría de series de tiempo.
} 
de 1946. Los autores visualizaron el ciclo económico como un fenómeno que permite la construcción de una cronología generalmente aceptada para los "turning points" o puntos de inflexión que experimenta la actividad económica. En el libro, los autores definen el ciclo de la siguiente manera:

El ciclo es un tipo de fluctuación muy común en el nivel de actividad económica agregada de los países que organizan las actividades productivas principalmente a través de firmas: un ciclo está compuesto de expansiones que suceden simultáneamente en diferentes actividades, seguidas generalmente por recesiones, contracciones y recuperaciones que se funden entre la fase de expansión del siguiente ciclo; esta secuencia de cambios es recurrente pero no periódica; la duración del ciclo económico varía desde un año a diez o 12 años; los cuales no se pueden dividir en ciclos más cortos de carácter similar con amplitudes aproximadamente similares (Burns \& Mitchell, 1946, p. 3).

La definición de ciclo también fue importante por la noción de comovimiento entre las series de tiempo macroeconómicas cuando ellos definen el concepto de "puntos de inflexión" (turning points), es decir, cuando los puntos de inflexión (picos y valles) en varios indicadores de la economía agregada están altamente correlacionados o van de la mano en un corto periodo de tiempo con el ciclo económico.

En este trabajo se adopta el enfoque de series de tiempo para la estimación del ciclo del producto interno bruto-PIB-del sector agropecuario durante el periodo comprendido entre el primer trimestre de 1976 hasta el último trimestre de 2013 7 .

En la actualidad, los trabajos empíricos relacionados con la estimación del ciclo a partir de un enfoque de series de tiempo parten del supuesto de que las variables siguen procesos lineales estocásticos. La ventaja de este enfoque es que permite integrar la teoría económica y la econometría de series de tiempo a un conjunto de información disponible en el sector agropecuario para variables como el PIB, empleo, desempleo, precios del sector, importaciones, exportaciones, entre otros, como más adelante se mostrará.

Con el fin de poner en práctica el enfoque de series de tiempo se supone que el nivel de actividad económica en el sector agropecuario se encuentra afectado por dos tipos de choques. En el caso colombiano, Albert Berry (1970) valida dicho supuesto al mencionar que las fluctuaciones del PIB a lo largo de

Si bien en este trabajo no se realiza una cronología del ciclo del PIB agropecuario tal como lo sugiere la metodología del National Bureau of Economic Research -NBER- de los Estados Unidos, el interés en este trabajo es descomponer la serie y estimar los efectos de los choques de demanda y oferta sobre el PIB del sector. 
una tendencia a largo plazo han estado asociadas a los ciclos de comercio internacional, aunque el desempeño económico se comportó relativamente bien cuando no existió el boom de exportaciones de café.

La brecha de la tasa de crecimiento con relación a los periodos de fuerte y débil desempeño de la balanza comercial ha sido dramática. El autor concluye que los choques de precios internacionales de café y el consiguiente boom exportador en este producto han sido una de las perturbaciones más importantes en las fluctuaciones del PIB del sector agropecuario a lo largo de la década de los 70 .

Por consiguiente, se asume que el primer tipo de shock genera efectos de naturaleza permanente sobre el PIB del sector y se llamará "shock permanente", como por ejemplo las mejoras en la productividad en el sector y los cambios a lo largo del tiempo en la fuerza laboral, cuya trayectoria ha estado marcada por flujos migratorios del campo a la ciudad (Londoño, 1984).

El segundo tipo de shock tiene efectos de naturaleza transitoria sobre el nivel de actividad agropecuaria, que desaparecen a lo largo del tiempo, los cuales pueden consistir en malas cosechas por efectos climáticos adversos, efectos demográficos que afectan el consumo de alimentos ${ }^{8}$, entre otros factores.

\section{La descomposición tradicional}

El enfoque tradicional del ciclo económico caracteriza el comportamiento de la economía a través de fluctuaciones del PIB agropecuario alrededor de una tendencia suave a largo plazo, que puede ser afectada por fluctuaciones cíclicas o de corta duración. Con el fin de estimar la tendencia a largo plazo, inicialmente se utiliza el método más simple, que consiste en evaluar mediante una regresión lineal la tasa de crecimiento exponencial del logaritmo del PIB agropecuario en función de una tendencia lineal ${ }^{9}$. Con ese propósito, se sigue la metodología de Okun (1962) como un enfoque alternativo para capturar el crecimiento de la productividad y el estado de la tecnología, factores que pueden afectar de manera importante la producción a largo plazo.

Okun definió la tendencia o el "producto potencial" como el nivel de producto que podría prevalecer o mantenerse si la tasa de desempleo fuera de $4 \%$, y realizó una regresión que toma como variable dependiente las primeras diferencias del producto en función de las primeras diferencias de la tasa de desempleo, y

\footnotetext{
$8 \quad$ De acuerdo con el Banco Mundial, el fenómeno inflacionario en el precio de alimentos en el ámbito mundial suscitado a mediados del año 2008, estuvo determinado, entre otros factores, por el aumento en el nivel de demanda de países emergentes como la China, propiciado por el crecimiento poblacional y el aumento en el consumo de proteínas.

9 No obstante, factores por el lado de la oferta como el crecimiento de la productividad, al parecer, no quedan bien capturados con este método de estimación (Blanchard \& Fischer, 1986).
} 
encontró que una reducción en la tasa de desempleo del orden de $1 \%$ estuvo asociada con un incremento en el PIB de $3 \%{ }^{10}$. Al replicar la metodología de Okun para los datos colombianos del sector agropecuario, para el periodo 1976 a 2013, se halló una relación de 1 a 3.2 , que coincide aproximadamente con la estimación encontrada originalmente por Okun. Lo que indica que por cada reducción en la tasa de desempleo en $1 \%$, el PIB del sector agropecuario se expandió en promedio al $3.2 \%$ a lo largo de estos 37 años. A continuación se muestra el resultado de la estimación:

$$
\Delta \ln P I B_{t}=0.28-\underbrace{0.032845}_{t=-3.11, p=0.002} * U_{t}
$$

Dicho hallazgo para el sector agropecuario implicaría, bajo condiciones ideales, que para mantener una senda de crecimiento del PIB más o menos cercana al potencial, el sector en promedio tuvo que haber crecido $3.2 \%$ durante este periodo, es decir 0.6 puntos porcentuales por encima del crecimiento promedio observado durante los últimos 37 años, que fue de $2.6 \%$. No obstante, en la sección anterior, el ejercicio de contabilidad del crecimiento mostró que parte de ese crecimiento del sector se apoyó en el crecimiento del producto por trabajador y fuertemente por el crecimiento de la PTF.

\section{El filtro de Hodrick y Prescott - HP-}

Debido a que uno de los objetivos primordiales de este artículo es la estimación del ciclo a partir de un enfoque de series de tiempo, en esta sección se describe de manera detallada la metodología del filtro de Hodrick y Prescott, la cual se caracteriza por analizar las propiedades estadísticas de las series de tiempo para el PIB del sector agropecuario.

Por otro lado, la estimación de la tendencia a largo plazo realizada en la sección anterior no da una idea precisa de lo que en la práctica sería esa tendencia a largo plazo que se asocia al PIB potencial en el sector. Para tal fin, se utiliza un método de filtrado que consiste en descomponer la serie del PIB agropecuario en la parte permanente (tendencia) y la parte transitoria (componentes cíclicos), mediante diferentes filtros.

La descripción del marco conceptual sobre el filtro de Hodrick y Prescott (1980) sigue de cerca la exposición realizada por Ahumada y Garegnani (1999), quienes parten del supuesto de que la serie $y_{t}$ está compuesta por la suma de un componente de crecimiento $g_{t} \mathrm{y}$ de un componente cíclico $c_{t}$ de manera que:

$$
y_{t}=g_{t}+c_{t}
$$

\footnotetext{
$\overline{10}$ A esta relación de 3 a 1 se la conoce como la "Ley de Okun" y en la actualidad ha sido blanco de grandes críticas, dado que los estudios empíricos para contrastarla sugieren más bien una regularidad empírica y no una ley en sentido estricto.
} 
El componente de crecimiento se determina a partir de la solución del siguiente problema:

$$
\operatorname{Min}\left\{\sum_{t=1}^{T} c_{t}^{2}+\lambda \sum_{t=1}^{T-1}\left[\left(g_{t}-g_{t-1}\right)-\left(g_{t-1}-g_{t-2}\right)\right]^{2}\right\}
$$

Donde el componente cíclico es la desviación de la serie observada con respecto de la tendencia a largo plazo, la cual será en promedio cercana a cero, a medida que se incrementa el horizonte de tiempo, y la suavización del componente de crecimiento se expresa mediante la suma de cuadrados de la segunda diferencia de la serie, es decir:

$$
\Delta^{2} g_{t}=(1-L)^{2} g_{t}=\left[\left(g_{t}-g_{t-1}\right)-\left(g_{t-1}-g_{t-2}\right)\right]
$$

Donde L denota el operador de rezago definido como $L x_{t}=\mathrm{x}_{t-1}$.

El parámetro $\lambda$ es un número positivo, el cual penaliza la variabilidad en el componente de crecimiento, de tal forma que entre más grande sea su valor, más suave será el componente $g_{t}$. Cuando $\lambda$ tiende a infinito, la primera diferencia $\Delta g_{t}=\left(g_{t}-g_{t-1}\right)$ tiende a una constante y la solución del problema se reduce a una estimación lineal de la tendencia estimada por mínimos cuadrados ordinarios (MCO). En este marco conceptual original, el valor a priori del parámetro de suavizamiento se obtiene asumiendo un modelo de probabilidad, en el cual:

$$
\begin{gathered}
c_{t} \sim N I\left(0, \sigma_{c}^{2}\right) \\
\Delta^{2} g_{t}=\varepsilon_{t} \sim N I\left(0, \sigma_{g}^{2}\right)
\end{gathered}
$$

El valor esperado de $g_{t}$, dado el conjunto de observaciones, es la solución del problema especificado por la ecuación [5] cuando $\lambda^{1 / 2}=\left(\sigma_{c} / \sigma_{g}\right)$. Hodrick y Prescott sugieren para datos trimestrales un parámetro de suavizamiento $\lambda^{1 / 2}=[5 /(1 / 8)]$ y $\lambda=1600$. Sin embargo, ellos reconocen la restricción impuesta por los supuestos y realizan un análisis de simulación para los valores de $\lambda$ en cada caso.

El enfoque original de HP amerita el análisis de tres aspectos, a saber: i) dada la ecuación [4], ningún componente irregular se asume en la descomposición de la serie y por tanto podría estar incorporado en el componente cíclico; ii) el problema de minimización expresado por la ecuación [5] y como consecuencia de i), supone que $c_{\mathrm{t}}$ es el residuo en la estimación del crecimiento (tanto el ciclo como el crecimiento no son observables); y iii) el valor de $\lambda$ no se determina, en principio, por optimización, sino más bien por una práctica empírica de los investigadores.

Un aspecto relevante del uso del filtro de HP se puede derivar a partir del análisis realizado por King y Rebelo(1993), quienes consideran el caso de "muestras infinitas", en donde el componente de crecimiento puede ser expresado de la siguiente forma: 


$$
g_{t}=\Sigma_{j=-\infty}^{\infty} w_{j} y_{t-1}=G(L) y_{t}
$$

Esto es, $g_{t}$ es un promedio móvil ponderado de la serie original y por lo tanto, el componente cíclico también es un promedio móvil de las series, es decir:

$$
c_{t}=[1-G(L)] y_{t}=C(L) y_{t}
$$

En donde $C(L)$ y $G(L)$ son filtros lineales.

Dado el conjunto de información de este problema de optimización y la muestra total de la serie, la condición de primer orden del problema [5] dado [4], es:

$$
\begin{gathered}
0=-2\left(y_{t}-g_{t}\right)+2 \lambda\left[\left(g_{t}-g_{t-1}\right)-\left(g_{t-1}-g_{t-2}\right)\right]-4 \lambda\left[\left(g_{t+1}-g_{t}\right)-\left(g_{t}-g_{t-1}\right)\right] \\
+2 \lambda\left[\left(g_{t+2}-g_{t+1}\right)-\left(g_{t+1}-g_{t}\right)\right]
\end{gathered}
$$

La cual podemos reescribir como:

$$
F(L) g_{t}=y_{t}
$$

En donde $F(L)$ es el polinomio de rezagos y está dado por:

$$
\begin{gathered}
F(L)=\lambda L^{-2}-4 \lambda L^{-1}+(6 \lambda+1)-4 \lambda L+\lambda L^{2} \\
F(L)=\left[\lambda(1-L)^{2}\left(1-L^{-1}\right)^{2}+1\right] \\
F(L)=\left[\lambda \Delta^{4^{*}}+1\right]
\end{gathered}
$$

Donde el asterisco indica la segunda diferencia centrada del operador de rezago o de adelanto de la serie, de forma que:

$$
\Delta^{4 *}=L^{-2}-4 L^{-1}+6-4 L+L^{2}=\left[(1-L)^{2}\left(1-L^{-1}\right)^{2}\right]
$$

Por tanto,

$$
F(L)^{-1}=G(L) \text { y } C(L)=[F(L)-1] * F(L)^{-1}=\lambda \Delta^{4^{*}} /\left[\Delta^{4^{*}}+1\right]
$$

A partir de esta presentación, King y Rebelo concluyen que este filtro cíclico es capaz de exigir estacionariedad a cualquier proceso integrado de cuarto orden, dado que existen cuatro diferencias en el numerador.
Es importante resaltar que actualmente existe abundante literatura que describe las diferentes críticas del filtro de HP, relacionadas principalmente con la optimalidad del filtro que puede ser operativo 
en casos especiales en donde se quiere minimizar el cuadrado del error $y$, por otro lado, la posibilidad de estimar ciclos espurios cuando se filtran datos que presentan una representación de "paseo aleatorio" (Ravn \& Uhlig, 1997). Sin embargo, en este trabajo no nos concentraremos en estas críticas, al contrario, implementaremos los filtros disponibles en el paquete econométrico de STATA, pero sin desconocer las limitaciones que estos traen.
En la Figura 5 se presenta el resultado del ejercicio de descomposición de la serie del PIB mediante el filtro de Hodrick y Prescott (1980) ${ }^{11}$. El PIB del sector ha fluctuado alrededor de una tendencia suave a largo plazo, la cual podría contener componentes determinísticos y estocásticos y por construcción, este componente no es estacionario. Lo anterior implica que los choques exógenos por el lado de la oferta, como las innovaciones tecnológicas y de productividad, habrían tenido efectos permanentes sobre el nivel de producto agropecuario.

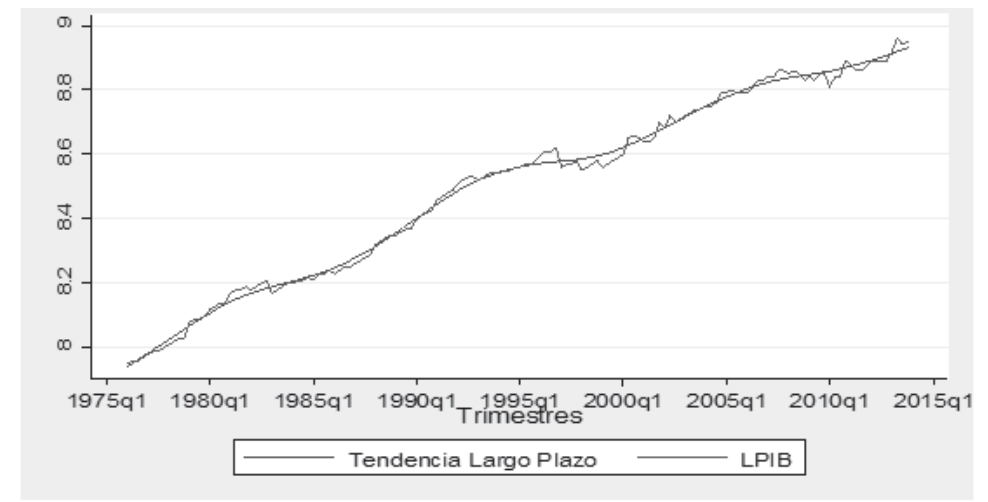

Figura 5. PIB agropecuario observado y tendencia a largo plazo.

Fuente: cálculos propios sobre cifras DANE - Sistema de Cuentas Nacionales.

$11 \quad$ Bajo este enfoque, el ciclo se obtiene por residuo, es decir, mediante la diferencia entre el valor observado del PIB y su tendencia a largo plazo, y su interpretación normalmente se da como la desviación en puntos porcentuales del componente observado con respecto al valor a largo plazo. 
La estimación del componente cíclico del PIB agropecuario mediante el filtro de HP que se muestra en la Figura 5 no da una idea de qué tan bien se ha estimado dicho componente. Con el fin de evaluar la estimación del componente cíclico, se puede obtener el periodograma, el cual es un estimador de la función de densidad espectral ${ }^{12}$ que muestra las frecuencias estándar divididas por $2 \pi$.

El programa STATA 13 permite graficar el periodograma con dos líneas verticales que cortan la frecuencia natural más baja a partir de ciclos estocásticos entre 6 y 32 periodos $^{13}$. El punto inferior de las líneas verticales se obtiene como $1 / 32=$ 0.03125 , y el punto superior se obtiene a partir de $1 / 6=0.16667$. Más adelante se presenta un análisis gráfico para evaluar el componente cíclico, mediante la estimación de un ciclo ideal que se compara con el ciclo resultante de aplicar el método de HP.

La Figura 6 muestra el periodograma del componentecíclico para el PIB agropecuario, en donde se observa una alta y baja periodicidad. Los puntos por encima del valor -6.00 a la izquierda del eje vertical derecho del gráfico, revelan que el filtro de HP no hizo un buen trabajo de filtrado para ciclos estocásticos de alta periodicidad, cuando se utiliza por defecto un valor del parámetro lambda igual a 1.600. Por otro lado, el periodograma revela que no existe una tendencia de los puntos ubicados a la derecha del eje vertical (puntos que se encuentran entre las líneas verticales) a concentrarse en el valor de -6.00 , lo que indica que el filtro HP no removió completamente el ciclo estocástico de baja periodicidad. Dicho resultado no es sorprendente, dado que el filtro de HP se caracteriza por ser un filtro de alta frecuencia.

12 En matemáticas y en física, la densidad espectral de una señal es una función matemática que nos informa de cómo está distribuida una variable aleatoria de dicha señal sobre las distintas frecuencias de las que está formada, es decir, su espectro.

13 Las líneas verticales del periodograma corresponden a los valores de 6 y 32 del componente del ciclo de negocios, utilizados originalmente por Burns y Mitchell (1946). 


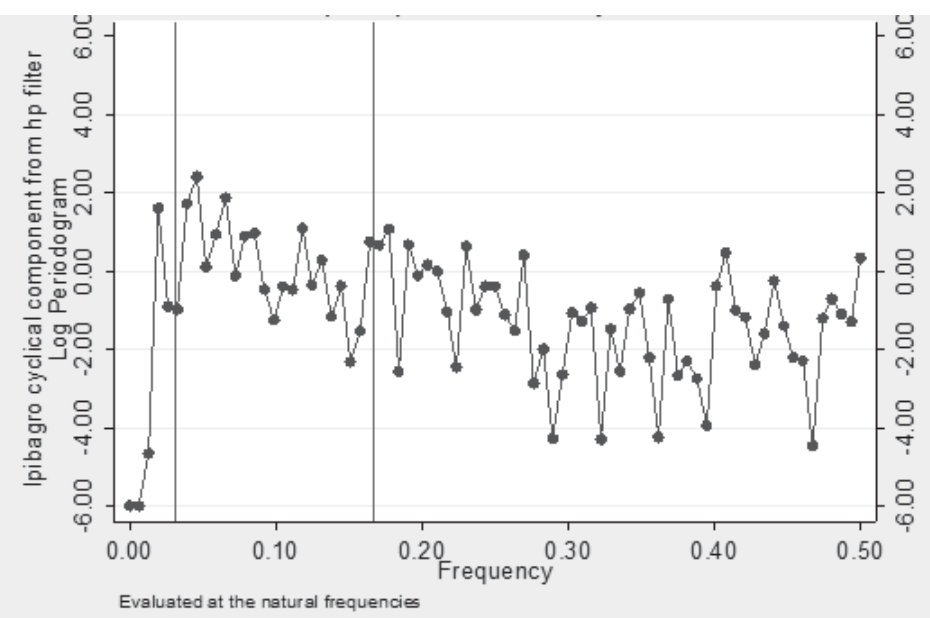

Figura 6. Sample spectral density function.

Fuente: cálculos propios sobre cifras DANE, Sistema de Cuentas Nacionales.

La Tabla 2 revela que el PIB del sector estuvo cerca de 12 años por encima de la tendencia a largo plazo y que la máxima desviación con respecto a la tendencia fue de 2.2 puntos porcentuales, que podría constituirse en el pico más alto antes de iniciarse la recesión del año 1999. No obstante, el nivel de producción se ubicó por debajo de la tendencia a largo plazo durante nueve años, en donde la desviación más baja con respecto al PIB tendencia fue de -2.3 puntos porcentuales, en un periodo que coincide con el punto más bajo de la producción alcanzado por el sector y que podría constituirse como un punto de giro en el nivel de actividad económica.
Con el fin de estimar la duración de los ciclos de una manera más precisa, se utilizó el enfoque propuesto por el Euro Area Business Cycle Dating Committee del Centre for Economic Policy Research (CEPR). Dicho organismo considera una recesión como un declive en el nivel de actividad económica, que se caracteriza usualmente por dos o más trimestres consecutivos de crecimiento negativo en el PIB y del empleo.

De acuerdo con la metodología del $\mathrm{CEPR}^{14}$, se registran tres ciclos completos para el sector agrícola con una duración de 16 trimestres (4 años) medidos de pico a pico, o de 15 trimestres $(3,8$

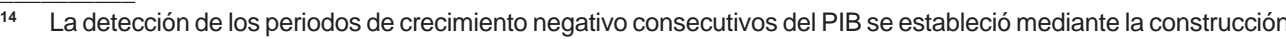
de una variable dummy que toma el valor de 1 , si la tasa de crecimiento del PIB agrícola fue negativa y cero en caso contrario.
} 
años) medidos de valle a valle. Las fases de expansión duran, en promedio, 29 trimestres ( 9,6 años), y las de contracción, 4 trimestres (1 año), lo que implica que los ciclos en el sector agrícola son asimétricos, dada la mayor duración de las expansiones con relación a las contracciones.

Los ciclos del PIB agrícola, comparados con los del PIB total, son más cortos y de menor frecuencia. En efecto, de acuerdo con Arango, Alfonso, Arias, Cangrejo y Pulido (2013), entre 1975 y 2011 se han presentado cuatro ciclos de negocios completos, los cuales duran en promedio 81 meses, es decir 6,8 años. Al igual que en el caso agrícola, los ciclos para el total de la economía son asimétricos, ya que las expansiones tardan, en promedio,
65 meses (5,4 años), mientras que las contracciones tardan 16 meses (1,3 años).

La Tabla 2 también incluye las políticas implementadas por el Gobierno tanto en la fase expansiva como en la recesiva ${ }^{15}$, catalogadas como procíclicas y contracíclicas, respectivamente. Se puede evidenciar que el Gobierno ha diseñado una política macroeconómica caracterizada por propiciar el desarrollo del sector, mediante una combinación de políticas anticíclicas efectivas con una estrategia activa de diversificación productiva. Así mismo, la política anticíclica reflejó el reto que implican las acentuadas fluctuaciones de los precios de productos básicos en el contexto internacional, especialmente los relacionados con los precios internacionales del café.

\footnotetext{
$\overline{15}$ La fase expansiva se determina a través de las desviaciones positivas del PIB con respecto a la tendencia a largo plazo, mientas que la fase recesiva son las desviaciones negativas con respecto a la tendencia a largo plazo.
} 
Tabla 2. Fases de la tasa de crecimiento del ciclo y políticas

\begin{tabular}{|c|c|c|c|}
\hline \multicolumn{4}{|c|}{ Por encima de la tendencia a largo plazo } \\
\hline Desde-hasta & Duración & Desviación* & Políticas procíclicas \\
\hline $\begin{array}{l}\text { 1.er trimestre de } 1979 \\
\text { - 4. }{ }^{\circ} \text { trimestre de } 1982\end{array}$ & 4 años & 1.5 & $\begin{array}{l}\text { Oferta: } \\
\text { - Creación del Consejo Nacional } \\
\text { de Investigación y Transferencia } \\
\text { de Tecnología, con el fin de fijar } \\
\text { políticas de investigación a medio } \\
\text { y largo plazo. } \\
\text { - Se establece el Fondo Nacional de } \\
\text { Investigaciones Agrope-cuarias y } \\
\text { de Recursos Naturales Renova- } \\
\text { bles, “como mecanismo financie- } \\
\text { ro que permita el flujo y el manejo } \\
\text { de recursos para el financiamiento } \\
\text { de actividades prioritarias seña- } \\
\text { ladas por el Consejo”. }\end{array}$ \\
\hline $\begin{array}{l}\text { 1.er trimestre de } 1988 \\
-4 .^{\circ} \text { trimestre de } 1988\end{array}$ & 1 año & 0.6 & $\begin{array}{l}\text { Oferta: } \\
\text { - Crédito de fomento a la produc- } \\
\text { ción y explotación, con el fin de }\end{array}$ \\
\hline $\begin{array}{l}\text { 1.er trimestre de } 1991 \\
\text { - } 4 .^{\circ} \text { trimestre de } 1992\end{array}$ & 2 años & 1.8 & $\begin{array}{l}\text { ción del sector. } \\
\text { • Rehabilitación, complementación } \\
\text { y ampliación de distritos de riego. }\end{array}$ \\
\hline $\begin{array}{l}\text { 2. } .^{\circ} \text { trimestre de } 1995 \\
\text { - } \text {. }^{\circ} \text { trimestre de } 1996\end{array}$ & $\begin{array}{c}1 \text { año y } 9 \\
\text { meses }\end{array}$ & 2.2 & $\begin{array}{l}\text { Oferta: } \\
\text { - Con el fin de propiciar el desarro- } \\
\text { llo rural campesino, se otorgaron }\end{array}$ \\
\hline $\begin{array}{l}\text { 2. }{ }^{\circ} \text { trimestre de } 2002 \\
\text { - 3.er trimestre } 2003\end{array}$ & $\begin{array}{c}1 \text { año y } 6 \\
\text { meses }\end{array}$ & 0.7 & $\begin{array}{l}\text { - Programas de crédito para pe- } \\
\text { queños y medianos agricultores } \\
\text { e incentivos a la capitalización } \\
\text { rural. }\end{array}$ \\
\hline $\begin{array}{l}\text { 3.er trimestre de } 2006 \\
\text { - 3er trimestre de } 2008\end{array}$ & $\begin{array}{c}2 \text { años y } 3 \\
\text { meses }\end{array}$ & 1.8 & $\begin{array}{l}\text { Oferta: } \\
\text { - Estímulo al desarrollo de proyec- } \\
\text { tos productivos y de generación }\end{array}$ \\
\hline $\begin{array}{l}\text { 1.er trimestre de } 2013 \\
\text { - } 4 .^{\circ} \text { trimestre de } 2013\end{array}$ & 1 año & 2.0 & $\begin{array}{l}\text { - Incentivos al desarrollo de in- } \\
\text { fraestructura física y social, rela- } \\
\text { cionada con la mejora en infraes- } \\
\text { tructura de transporte. }\end{array}$ \\
\hline
\end{tabular}




\begin{tabular}{|c|c|c|c|}
\hline \multicolumn{4}{|c|}{ Por debajo de la tendencia a largo plazo } \\
\hline Desde-hasta & Duración & Desviación* & Políticas contracíclicas \\
\hline $\begin{array}{l}\text { 1.er trimestre de } 1985 \\
\text { - } \text {. }^{\circ} \text { trimestre de } 1987\end{array}$ & 3 años & -1.2 & \multirow{2}{*}{$\begin{array}{l}\text { Demanda: } \\
\text { - Reactivación demanda de bienes } \\
\text { agropecuarios. } \\
\text { - Flujo recursos de crédito al sector } \\
\text { por PROEXPORT. } \\
\text { Oferta: } \\
\text { - Investigación y transferencia de } \\
\text { tecnología. } \\
\text { - Reactivación oferta y aumento } \\
\text { de productividad por racionali- } \\
\text { zación de costos insumos (ferti- } \\
\text { lizantes, semillas, maquinaria y } \\
\text { agroquímicos). }\end{array}$} \\
\hline $\begin{array}{l}\text { 1.er trimestre de } 1989 \\
\text { - } 4 .^{\circ} \text { trimestre de } 1990\end{array}$ & 2 años & -0.9 & \\
\hline $\begin{array}{l}\text { 3.er trimestre de } 1997 \\
\text { - 1.er trimestre de } 2000\end{array}$ & $\begin{array}{l}2 \text { años y } \\
\text { medio }\end{array}$ & -2.3 & $\begin{array}{l}\text { Oferta: } \\
\text { - Flujos crédito a través de Caja } \\
\text { Agraria para producción agro- } \\
\text { pecuaria. } \\
\text { - Estímulos a oferta agropecuaria } \\
\text { a través de organizar cadenas } \\
\text { productivas. } \\
\text { - Inversión rural y subsidios a } \\
\text { inversión en tecnología y bienes } \\
\text { capital. } \\
\text { - Fortalecimiento de C \& T. } \\
\text { - Desarrollo núcleos productivos } \\
\text { para integración sector rural. }\end{array}$ \\
\hline $\begin{array}{l}\text { 3.er trimestre de } 2005 \\
\text { - 2. }{ }^{\circ} \text { trimestre de } 2006\end{array}$ & 1 año & -0.7 & \multirow{2}{*}{$\begin{array}{l}\text { Oferta: } \\
\text { - Profundizan una serie de acuer- } \\
\text { dos comerciales (Canadá, UE, } \\
\text { TLC USA) con el fin de dinami- } \\
\text { zar oferta exportable sector. } \\
\text { - Frente externo reforma estructu- } \\
\text { ral arancelaria (aranceles planos } \\
\text { y eliminar la dispersión). }\end{array}$} \\
\hline $\begin{array}{c}\text { 2. }^{\circ} \text { trimestre de } 2011 \\
-4 .^{\circ} \text { trimestre de } 2012\end{array}$ & $\begin{array}{c}1 \text { año y } 9 \\
\text { meses }\end{array}$ & -1.3 & \\
\hline
\end{tabular}

Fuente: cálculos propios sobre cifras DANE - Sistema de Cuentas Nacionales.

$(*)$ : desviación medida en puntos porcentuales. 


\section{CHOQUES EXÓGENOS Y CICLO DEL PIB AGROPECUARIO}

Una vez estimado el componente cíclico, que por construcción es una serie estacionaria, se construye un modelo ARIMA de series de tiempo, con el fin de analizar el comportamiento estocástico del ciclo cuando es afectado por choques exógenos y determinar la duración en el tiempo del efecto. En el anexo 1 del trabajo se presenta una serie de pruebas de raíz unitaria, para analizar la estacionariedad del componente cíclico y la tendencia.
En estricto sentido, la metodología que se sigue es la propuesta por Box y Jenkins (1976), con el fin de estimar la función de impulso respuesta del proceso para medir los multiplicadores derivados de los choques sobre el ciclo del PIB agropecuario. Al utilizar el correlograma de la serie de ciclo y los criterios de información ${ }^{16}$, se concluye que el comportamiento del ciclo del PIB queda bien capturado por un modelo $\operatorname{ARMA}(2,4)$, de la forma:

$$
\begin{gathered}
\text { CicloPIB }_{t}=1.58 * \text { CicloPIB }_{t-1}-0.88 * \text { CicloPIB }_{t-2}+ \\
\varepsilon_{t}-1.0 * \varepsilon_{t-1}+0.39 * \varepsilon_{t-2}-0.04 * \varepsilon_{t-3}+0.4 * \varepsilon_{t-4} \\
\sigma_{\varepsilon}=0.0124, p=0.000
\end{gathered}
$$

El término $\varepsilon_{t}$ captura el componente de media móvil (MA), el cual se asocia al choque aleatorio y es por construcción, la parte de la desviación del PIB agropecuario con respecto a la tendencia que no se puede predecir a partir del comportamiento pasado del PIB; es decir, serían todas las sorpresas que han afectado a la actividad agropecuaria, incluyendo factores climáticos y el efecto de la política económica. El modelo se evaluó por máxima verosimilitud y permitió una estimación de la desviación estándar igual a $1.2 \%$, y refleja los movimientos trimestrales del PIB que no pueden predecirse a partir del pasado de la serie. Dicha estimación, al igual que todos los parámetros de los componentes AR y MA, resultó ser estadísticamente significativa y diferente de cero.

Una forma de interpretar la ecuación [18] es explotar la estimación de los coeficientes que representan el promedio móvil del proceso y utilizar la función de impulso respuesta, con el fin de describir los efectos dinámicos de un shock $\varepsilon_{t}$ sobre el PIB a lo largo del tiempo. La Figura 7 presenta el efecto dinámico de un choque exógeno, el cual se recoge en el término $\varepsilon_{t}$, sobre la trayectoria futura seguida por el PIB.

La función de impulso respuesta traza la respuesta dinámica del PIB agropecuario

\footnotetext{
16 $\quad$ Básicamente se utilizaron los criterios de información de Akaike (AIC) y el bayesiano de Schwartz (SBIC) para determinar el modelo más parsimonioso o menos sobreparametrizado.
} 
a un choque bajo el supuesto de un modelo con tendencia estacionaria. En primer lugar, el efecto del choque genera una caída después del primer trimestre oscilando hasta el sexto trimestre (año y medio). A partir de este periodo, el efecto se diluye lentamente en el tiempo con un efecto latente del orden del $60 \%$, del choque inicial.

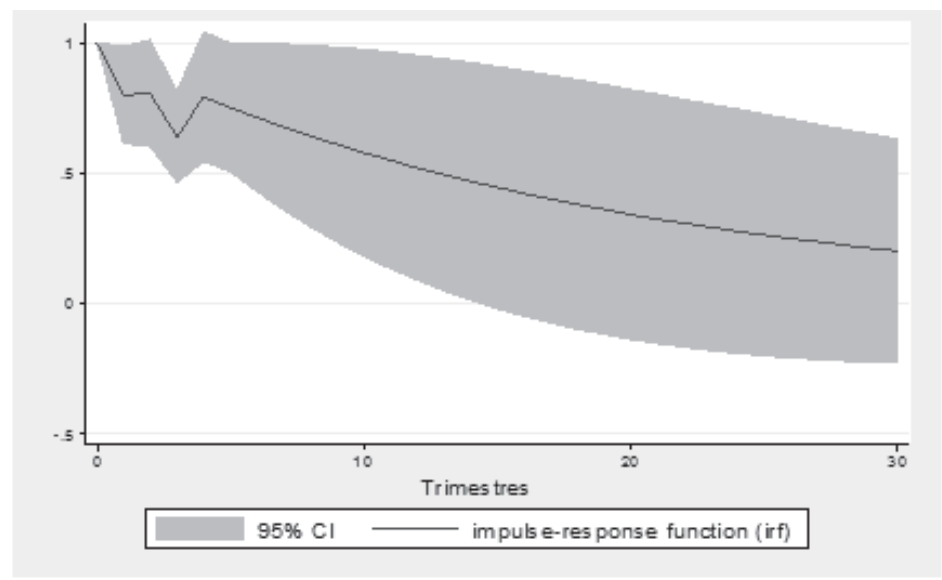

Figura 7. Función de impulso respuesta del PIB agropecuario.

Fuente: Cálculos propios sobre cifras DANE, Sistema de Cuentas Nacionales.

\section{Descomposición a la Campbell y Mankiw}

Una visión más reciente en torno al comportamiento del ciclo económico afirma que todas las fluctuaciones son el resultado de los efectos dinámicos que generan los choques permanentes de una serie, es decir, que el producto observado y la tendencia son iguales. Por tanto, eliminar la tendencia o suavizarla no tendría sentido económico, y el comportamiento del producto debe asociarse más a un proceso no estacionario en el cual todos los choques son de naturaleza permanente.
Campbell y Mankiw (1987a) construyeron a partir del trabajo de Nelson y Plosser (1982) un modelo ARIMA para describir el comportamiento del logaritmo del PIB trimestral en los Estados Unidos en época de posguerra, con el fin de mostrar al final que la tasa de crecimiento (no el nivel) del PIB sigue un proceso estacionario ARMA.

Con esta idea en mente, se procedió a implementar dicho procedimiento a partir de la modelación ARIMA propuesta por Box y Jenkins. La etapa de identificación 
sugiere que el comportamiento de la tasa de crecimiento del PIB agropecuario se captura mejor mediante un modelo
ARMA (1,2). El modelo estimado se presenta a continuación:

$$
\begin{gathered}
\Delta \ln P I B_{t}=0.026+0.41 * \Delta \ln P I B_{t-1}+\varepsilon_{t}+0.18 * \varepsilon_{t-1}+0.66 * \varepsilon_{t-2} \\
\sigma_{\varepsilon}=0.017, p=0.000
\end{gathered}
$$

En donde $\Delta \ln P I B_{t}=\ln P I B_{t}-\ln P I B_{t-1}$, es la tasa de crecimiento del PIB medida como diferencia de logaritmos. Al igual que el anterior modelo, todos los coeficientes estimados son estadísticamente significativos y diferentes de cero a un nivel de significancia del $1 \%$. El valor estimado del intercepto en la ecuación [19] es positivo y su magnitud refleja que el producto agropecuario ha mantenido una tasa de crecimiento promedio del orden de $2.6 \%$ durante estos últimos 37 años, resultado que ya se había analizado en la sección del análisis de contabilidad de crecimiento para el sector. La desviación estándar estimada del modelo es estadísticamente significativa $y$ en magnitud es mucho mayor que el modelo anterior.

La respuesta dinámica del PIB agropecuario a un choque aleatorio equivalente a una desviación estándar de $1.7 \%$ (se presenta en la Figura 8 ), que no es más que la función de impulso respuesta. La figura del efecto dinámico de un shock sobre el PIB muestra un salto del producto después de dos trimestres y después desciende rápidamente hacia el valor de cero. De esta manera, el shock inicial equivalente a una desviación estándar de 1,7 \%, disminuye el crecimiento del PIB después del primer trimestre y aumenta el segundo trimestre. Después de cuatro trimestres, todavía existe un $40 \%$ del choque inicial y después de ocho trimestres (dos años) prácticamente el efecto del choque ha desaparecido por completo. Note que la tasa de convergencia a cero de la tasa de crecimiento del PIB es alta, lo cual se refleja en el hecho de que la serie sea estacionaria.

El enfoque de Campbelly Mankiw permite una descripción del movimiento del PIB como resultado de una acumulación de choques a lo largo del tiempo, cada uno de los cuales es un promedio positivo, cuando el intercepto en la ecuación se tiene en cuenta, y tiene efectos de naturaleza transitoria si la serie es estacionaria, como es el caso de la tasa de crecimiento del PIB. Las disminuciones en la tasa de crecimiento del PIB son el resultado de choques negativos y los periodos de expansión son el resultado de grandes choques positivos. La implicación de lo anterior, equivale a decir que las condiciones de la senda de crecimiento del PIB agropecuario colombiano se han modificado sustancialmente, en parte por las ganancias en productividad de los recursos productivos y en parte, por el 
efecto de las políticas de oferta y demanda diseñadas dentro del sector, como se verá más adelante. Así mismo, y como lo muestra el ejercicio de impulso respuesta, los choques más significativos para el sector en términos de política han sido los que provienen del lado de la oferta, los cuales se han manifestado por las políticas de innovación e investigación propiciadas por las distintas administraciones.

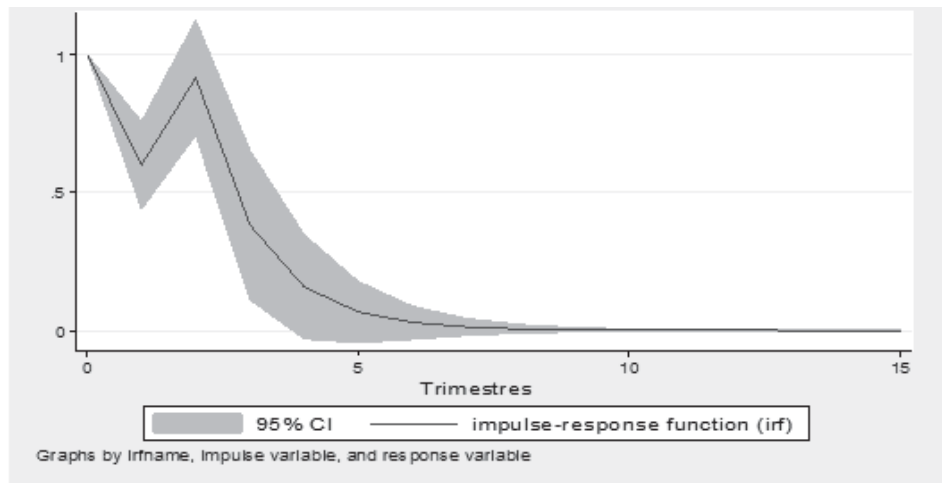

Figura 8. Función del impulso respuesta del PIB agropecuario.

Fuente: cálculos propios sobre cifras DANE, Sistema de Cuentas Nacionales.

\section{COMOVIMIENTOS DEL PIB AGROPECUARIO Y VARIABLES DE OFERTA Y DEMANDA}

En esta sección se analiza el comportamiento de variables de oferta y demanda y el PIB del sector agrícola, teniendo en cuenta las correlaciones de forma contemporánea y rezagada entre los residuos estimados de los modelos univariados ARIMA para el PIB y el resto de variables, con el fin de ofrecer la siguiente interpretación: una correlación contemporánea alta y positiva entre las innovaciones ${ }^{17}$ del consumo aparente del sector y las innovaciones del PIB indican que los movimientos inesperados (sorpresas) del PIB, es decir, movimientos que no se pueden predecir a partir del comportamiento pasado del producto están tradicionalmente asociados con movimientos positivos en el consumo; en otras palabras, la variable es procíclica. Es importante resaltar que la correlación no implica causalidad, sino por el contrario está mostrando el grado de asociación estadística entre las dos variables.

\footnotetext{
$\overline{17} \quad$ La correlación contemporánea tiene que ver con el grado de asociación estadística entre las variables en el mismo periodo, mientras que la correlación con rezagos analiza el grado de asociación entre las variables pero en distintos periodos de tiempo.
} 
La selección de las variables ${ }^{18}$ para el ejercicio de comovimientos obedece en primer lugar a la disponibilidad de datos y en segundo lugar, como es común en el análisis macroeconómico a partir del modelo de demanda y oferta agregada, busca indicadores que describan la dinámica de la oferta y la demanda. Por ejemplo, algunos indicadores por el lado de la oferta normalmente son los salarios reales, los precios de la materia prima (petróleo) y commodities propios del sector como el precio de la palma de aceite, entre otros, que sirven como insumos en la producción. Por su parte, como indicadores de la demanda "agregada" en el sector, se utiliza el consumo aparente, exportaciones de café por su fuerte dinámica comercial en el contexto internacional, las exportaciones totales y el saldo de la balanza comercial del sector.

\section{Efecto de variables de demanda}

Con el fin de analizar qué tan elásticos son los componentes de demanda al ciclo del PIB total en el sector agropecuario, se seleccionaron algunas variables por el lado de la demanda del sector agrícola, tales como el consumo aparente del sector ${ }^{19}$, exportaciones totales del sector, exportaciones de café y la balanza comercial agropecuaria.

La Tabla 3 presenta los resultados de un modelo ARIMA para el logaritmo del PIB, el consumo aparente, la balanza comercial agropecuaria, las exportaciones totales de café y las exportaciones totales del sector agrícola.

La Tabla 3 también presenta la correlación entre las innovaciones (residuos estimados) del PIB y las innovaciones de los componentes del consumo aparente, exportaciones totales, exportaciones de café y balanza comercial del sector, desde los rezagos -3 hasta el adelanto 3 , es decir, la idea es ver de manera dinámica la correlación en el tiempo de las innovaciones.

\footnotetext{
18 En los anexos 2 y 3 se encuentra un conjunto de estadísticas descriptivas para las variables analizadas, así como las fuentes y las unidades de medida.

19 Definido como PIB agropecuario $\left(P I B A_{t}\right)$ más las importaciones totales del sector $\left(M A_{t}\right)$ y descontando las exportaciones $\left(X A_{t}\right)$, es decir $C A_{t}=P I B A_{t}+M A_{t}-X A_{t}$
} 
Tabla 3. Comovimientos del PIB agrícola y sus componentes: correlaciones entre innovaciones ${ }^{1}$

\begin{tabular}{|l|r|r|r|r|r|r|r|}
\hline & \multicolumn{7}{|c|}{ Innovaciones del PIB en el periodo ${ }^{2}$ : } \\
\cline { 2 - 8 } Innovaciones en el periodo cero de: $^{*}$ & $-\mathbf{3}$ & $\mathbf{- 2}$ & $\mathbf{- 1}$ & $\mathbf{0}$ & $\mathbf{1 +}$ & $\mathbf{2 +}$ & $\mathbf{3 +}$ \\
\hline Consumo Aparente $^{\mathbf{3}}$ & 0,057 & $-0,059$ & 0,174 & 0,376 & $-0,007$ & $-0,017$ & $-0,035$ \\
\hline Exportaciones totales Sector $^{\mathbf{4}}$ & $-0,067$ & $-0,050$ & $-0,052$ & 0,086 & $-0,017$ & 0,009 & $-0,098$ \\
\hline Exportaciones de Café $^{5}$ & $-0,072$ & $-0,061$ & $-0,115$ & 0,081 & 0,040 & 0,001 & $-0,110$ \\
\hline Balanza Comercial Agropecuaria $^{\mathbf{6}}$ & $-0,070$ & $-0,051$ & $-0,005$ & 0,120 & $-0,008$ & $-0,021$ & $-0,107$ \\
\hline
\end{tabular}

\section{Notas:}

1. Residuos estimados a partir de un modelo ARIMA para el PIB y sus componentes. Datos trimestrales a pesos del 2005. Tasas de crecimiento anual entre 1976 -I y 2013 - IV.

2. $\Delta \mathrm{y}_{\mathrm{t}}=0.026+0.415^{*} \Delta \mathrm{y}_{\mathrm{t}-1+\mathrm{et}}+0.184^{*} \mathrm{et}_{-1}+0.668^{*} \mathrm{et}_{-2,} \mathrm{~s}=0.017$. Donde $\mathrm{y}_{\mathrm{t}}$ es el logarítmo del PIB y s el error estándar estimado.

3. $\Delta \mathrm{ca}_{\mathrm{t}}=0.0263+\mathrm{e}_{\mathrm{t}}+0.793 * \mathrm{et}_{-1}+0.804 * \mathrm{et}_{-2}+0.858 * \mathrm{et}_{-3}, \mathrm{~s}=0.034$. Donde $\mathrm{ca}_{\mathrm{t}}$ es el logarítmo del consumo aparente y s el error estándar estimado.

4. $\Delta$ exptot $_{\mathrm{t}}=0.0266+0.833 *$ ? $\operatorname{exptot}_{\mathrm{t}-1}+\mathrm{e}_{\mathrm{t}}-0.284{ }_{\mathrm{et}-1}-0.72{ }^{*}{ }_{\mathrm{et}-4}, \mathrm{~s}=0.174$. Donde exptot $\mathrm{t}_{\mathrm{t}}$ es el logarítmo de las exportaciones totales y s el error estándar estimado.

5. $\Delta \operatorname{expcaf}_{\mathrm{t}}=0.0045+0.880 * \Delta \operatorname{expcaf}_{\mathrm{t}-1}+\mathrm{e}_{\mathrm{t}}-0.298 * \mathrm{e}_{\mathrm{t}-1}-0.684 * \mathrm{e}_{\mathrm{t}-4}, \mathrm{~s}=0.231$. Donde expcaf $\mathrm{t}_{\mathrm{t}}$ es el logarítmo de las exportaciones de café y s el error estándar estimado.6. ?bct $=0.0274+$ et $+0.686 *$ et- 1 $+0.678 *$ et- $2+0.689 *$ et- $3, \mathrm{~s}=0.223$. Donde bct es el logarítmo de la balanza comercial y s el error estándar estimado.

Fuente: cálculos propios sobre cifras DANE, Sistema de Cuentas Nacionales y DANE-DIAN.

Las estimaciones presentadas en la Tabla 3 confirman que las variables que capturan la demanda del sector (consumo aparente, exportaciones totales, exportaciones de café y balanza comercial del sector) se mueven en igual dirección que el PIB, es decir, la correlación entre las variables en el periodo 0 y el PIB es positiva y estadísticamente significativa. La correlación contemporánea (cuando se comparan las variables en el mismo periodo) entre las innovaciones del PIB y cada uno de los componentes es grande y significativa, mientas que las correlaciones entre las mismas variables en diferentes periodos (rezagos y adelantos) son más pequeñas, excepto para el consumo aparente y la balanza comercial agropecuaria, y no son estadísticamente significativas.

A partir de la información reportada en la tabla también se puede evaluar el coeficiente de una regresión estimada por MCO de la innovación de cada componente en función de la innovación del PIB, cuyo resultado se presenta en la Tabla $3^{20}$.

$20 \quad$ El coeficiente de regresión así estimado, es igual al coeficiente de correlación multiplicado por la relación de la desviación estándar de la innovación en el componente específico y la desviación estándar de la innovación en el PIB, es decir $\hat{\beta}=\left(\sigma_{x i} / \sigma_{P I B}\right) * \hat{p}_{x}$, PIB. Donde $\hat{\beta}$ es el coeficiente de regresión, $\sigma_{x i}$ la desviación estándar de cada componente, $\sigma_{P I B}$ la desviación estándar del PIB y $\hat{p}_{x}$, PIB el coeficiente de correlación estimado entre la innovación de cada componente y la innovación del PIB. 
Tabla 4. Elasticidad de los componentes de la demanda con respecto al PIB

\begin{tabular}{|l|c|c|c|c|}
\hline & \multicolumn{4}{|c|}{ Coeficientes } \\
\hline \multicolumn{1}{|c|}{ Componente } & Correlación $(\rho)$ & $\begin{array}{c}\text { Desviación } \\
\text { Stándar } \\
\text { Componente }\left(\sigma_{\mathrm{xi}}\right)\end{array}$ & $\begin{array}{c}\text { Desviación } \\
\text { Stándar PIB } \\
\left(\sigma_{\mathrm{PIB}}\right)\end{array}$ & $\begin{array}{c}\text { Elasticidad } \\
\hat{\beta}=\left(\sigma_{\mathrm{xi}} / \sigma_{\mathrm{PIB}}\right)^{*} \rho\end{array}$ \\
\hline Consumo Aparente & 0,3766 & 0,034 & 0,017 & 0,738 \\
\hline Exportaciones totales sector & 0,0869 & 0,175 & 0,017 & 0,873 \\
\hline Exportaciones de café & 0,0813 & 0,231 & 0,017 & 1,080 \\
\hline Balanza Comercial Agropecuaria & 0,1203 & 0,223 & 0,017 & 1,542 \\
\hline
\end{tabular}

Fuente: Cálculos propios a partir de la matriz de correlaciones. Cifras DANE y DANE - DIAN.

Es importante resaltar que dicho coeficiente no se interpreta como una relación causal y por consiguiente hay que tener cuidado en su interpretación. El coeficiente estimado da una idea de cuánto se mueve cada componente de demanda con respecto al PIB y por tanto refleja la elasticidad ${ }^{21}$ de cada innovación de la demanda con respecto a la innovación del PIB. De acuerdo con la Tabla 4, la elasticidad del consumo aparente con respecto al PIB es igual 0,73, que es mucho menor que $1, y$ refleja que un aumento de un punto porcentual en la innovación del PIB generó un aumento menos que proporcional en el consumo aparente.

La elasticidad de las exportaciones totales del sector agrícola fue de 0.87 , que también resultó ser menor a la unidad, lo que indica que un aumento de un punto porcentual en la innovación del PIB genera en promedio un incremento menos que proporcional en las exportaciones. Por su parte, la elasticidad de las exportaciones de café es igual a 1 , lo que señala una elasticidad unitaria de las exportaciones de café con respecto al PIB. En particular, las recesiones en el sector agrícola han estado acompañadas de caídas de igual magnitud en las exportaciones de café.

La elasticidad de la balanza comercial del sector con respecto a las innovaciones del PIB fue de 1.5, que es mucho mayor que uno, lo que implica que un incremento de un punto porcentual en la innovación del PIB produjo en promedio un incremento más que proporcional de 1.5 puntos porcentuales en el saldo de la balanza comercial. Esta estimación muestra que en las épocas de auge o expansión en donde el PIB observado del sector se ubicó por encima de la tendencia a largo plazo, como por ejemplo el periodo de

21 Los comovimientos en el análisis de ciclos permiten determinar la correlación entre el ciclo del PIB y el ciclo de cada uno de los componentes de la demanda agregada, con el fin de establecer cómo responden los componentes de demanda frente a cambios del componente ciclo del PIB, es decir, se quiere conocer cómo se mueven las variables de demanda con respecto al ciclo de la economía. 
1979 a 1982, que coincide con el periodo de bonanza cafetera y de altos precios internacionales, el balance externo del sector presentó superávits significativos.

La fuerte correlación entre el PIB, el consumo aparente, las exportaciones del café y la balanza comercial, es uno de los mejores hechos estilizados que han caracterizado a las fluctuaciones económicas en el sector agrícola en Colombia durante los últimos 37 años. Específicamente, la covarianza entre el consumo aparente y el PIB del sector es acorde con la idea de una propensión marginal al consumir que es positiva pero menor que la unidad.

\section{Comovimientos del PIB y precios}

La Tabla 5 describe los comovimientos entre el PIB y los precios internacionales de algunos commodities del sector, estimados mediante modelos ARIMA para el logaritmo del precio internacional del café, del precio internacional del petróleo, de la palma de aceite, del banano, del azúcar y del tabaco.

Tabla 5. Comovimientos del PIB agrícola y precios: correlaciones entre innovaciones ${ }^{1}$

\begin{tabular}{|l|c|c|c|c|c|c|c|}
\hline Innovaciones del PIB en el periodo $^{2}:$ & & & & & & & \\
\hline Innovaciones en el periodo cero de: & $\mathbf{- 3}$ & $\mathbf{- 2}$ & $\mathbf{- 1}$ & $\mathbf{0}$ & $\mathbf{1 +}$ & $\mathbf{2 +}$ & $\mathbf{3 +}$ \\
\hline Precio internacional de café $^{3}$ & $-0,1431$ & $-0,0048$ & $-0,0340$ & $-0,1009$ & 0,0365 & $-0,0072$ & $-0,0835$ \\
\hline Precio internacional del petróleo $^{4}$ & 0,0568 & 0,0755 & $-0,1017$ & 0,1588 & 0,1028 & 0,1335 & 0,0718 \\
\hline Precio internacional de palma $^{5}$ & $-0,1361$ & $-0,0197$ & 0,0944 & 0,0571 & $-0,0053$ & $-0,0403$ & $-0,0886$ \\
\hline Precio internacional de banano $^{6}$ & 0,0542 & 0,0914 & 0,0577 & $-0,1143$ & $-0,0203$ & 0,0214 & 0,1195 \\
\hline Precio internacional de azúcar $^{7}$ & $-0,1332$ & $-0,0613$ & 0,0961 & $-0,0349$ & $-0,0212$ & 0,0397 & 0,0120 \\
\hline Precio internacional de tabaco $^{8}$ & $-0,0130$ & 0,0762 & $-0,0565$ & $-0,1547$ & $-0,0589$ & 0,1118 & 0,0522 \\
\hline
\end{tabular}

Notas:

1. Residuos estimados a partir de un modelo ARIMA para el PIB y los precios. Datos trimestrales a pesos del 2005. Tasas de crecimiento anual entre 1976 -I y 2013 - IV.

2. $\Delta \mathrm{y}_{\mathrm{t}}=0.026+0.415 * \Delta \mathrm{y}_{\mathrm{t}-1}+\mathrm{e}_{\mathrm{t}}+0.184 * \mathrm{e}_{\mathrm{t}-1}+0.668 * \mathrm{e}_{\mathrm{t}-2}, \mathrm{~s}=0.017$. Donde $\mathrm{y}_{\mathrm{t}}$ es el logarítmo del PIB y s el error estándar estimado.

3. $\Delta \mathrm{pc}_{\mathrm{t}}=0.009+0.578 * \Delta \mathrm{pc}_{\mathrm{t}-1}+\mathrm{et}+0.845 * \mathrm{e}_{\mathrm{t}-1}, \mathrm{~s}=0.167$. Donde $\mathrm{pc}$ es el logarítmo del precio internacional del café y s el error estándar estimado.

4. $\Delta$ ppet $_{\mathrm{t}}=0.0575+0.519 * \Delta$ ppet $_{\mathrm{t}-1}+\mathrm{e}_{\mathrm{t}}-0.874 * \mathrm{e}_{\mathrm{t}-1}, \mathrm{~s}=0.161$. Donde ppet $\mathrm{t}_{\mathrm{t}}$ es el logarítmo del precio internacional del petróleo. y s el error estándar estimado.

5. $\Delta$ ppalm $t_{t}=0.0209+0.952 * \Delta$ ppalm $_{t-1}-0.148 * \Delta$ ppalm $_{\mathrm{t}-4}+$ et $+0.179 * \mathrm{e}_{\mathrm{t}-1}-0.777 * \mathrm{e}_{\mathrm{t}-4}, \mathrm{~s}=0.129$. Donde ppalmt es el logarítmo del precio de la palma y s el error estándar estimado.

6. $\Delta$ pban $_{t}=0.0312+0.518 * \Delta$ pban $_{t-1}+0.518 * \Delta$ pban $_{t-4}$ et $-0.134 * \mathrm{e}_{\mathrm{t}-1}-1.008 * \mathrm{e}_{\mathrm{t}-4}, \mathrm{~s}=0.144$. Donde pban es el logarítmo del precio internacional del banano y s el error estándar estimado.

7. $\Delta \mathrm{piaz}_{\mathrm{t}}=0.0119+1.023 * \Delta \mathrm{piaz}_{\mathrm{t}-1}-0.129 * \Delta \mathrm{piaz}_{\mathrm{t}-4}$ et $+0.062 * \mathrm{e}_{\mathrm{t}-1}-1.024 * \mathrm{e}_{\mathrm{t}-4}, \mathrm{~s}=0.152$. Donde piaz es el logarítmo del precio internacional del azúcar y s es el error estándar estimado.

8. $\Delta$ pint $_{\mathrm{t}}=0.0209+1.100 * \Delta$ pint $_{\mathrm{t}-1}-0.184 * \Delta$ pint $_{\mathrm{t}-4}$ et $+0.155^{*} \mathrm{e}_{\mathrm{t}-1}-0.919 * \mathrm{e}_{\mathrm{t}-4}, \mathrm{~s}=0.030$. Donde pint es el logarítmo del precio internacional del tabaco y s es el error estándar estimado.

Fuente: Cálculos propios sobre cifras del Banco Mundial y Federación Nacional de Cafeteros. 
Existe una baja correlación de forma contemporánea entre los precios internacionales y la producción del sector, aunque en la mayoría de los casos las correlaciones son positivas, excepto para el precio internacional del café y del petróleo. Este resultado podría sugerir que los episodios de sobreoferta a nivel mundial en la producción de café estuvieron asociados a caídas en el precio internacional del grano. En el caso del precio internacional del petróleo, que se considera como una materia prima, los incrementos en el precio generan un sobrecosto en el uso de insumos, lo cual podría haber generado caídas sensibles con la producción del sector. Por otro lado, las correlaciones de manera rezagada y adelantada de los precios con el PIB son muy bajas y estadísticamente no significativas.

La Tabla 6 presenta la estimación del coeficiente de regresión de cada uno de los precios de los productos con respecto al PIB, es decir, la elasticidad precio de la producción del sector agrícola.

Es interesante resaltar que la elasticidad precio del café con respecto al PIB del sector es unitaria, lo que implica que todos los choques de oferta se trasladaron a los precios en igual proporción y las dos variables se movieron en sentido inverso, es decir, los excesos de oferta implicaron precios bajos en la producción de café.

La elasticidad precio del petróleo con respecto a producto del sector es mayor que uno, lo que implica que los precios de la materia prima fueron muy elásticos ante los cambios en la producción del sector. En efecto, un incremento de un punto porcentual en la innovación del PIB generó en promedio un aumento más que proporcional del orden de 1,5 puntos porcentuales en el precio internacional del petróleo.

Tabla 6. Elasticidad precio de la producción

\begin{tabular}{|l|c|c|c|c|}
\hline \multicolumn{1}{|c|}{ Componente } & \multicolumn{3}{|c|}{ Coeficientes } \\
\hline Correlación $(\rho)$ & $\begin{array}{c}\text { Desviación } \\
\text { Stándar } \\
\text { Componente }\left(\sigma_{\mathrm{xi}}\right)\end{array}$ & $\begin{array}{c}\text { Desviación } \\
\text { Stándar PIB } \\
\left(\sigma_{\text {PIB }}\right)\end{array}$ & $\begin{array}{c}\text { Elasticidad } \\
\hat{\beta}^{\prime}\end{array}$ & $\left(\sigma_{\mathrm{xi}} / \sigma_{\text {PIB }}\right) * \rho$ \\
\hline Precio internacional del café & $-0,10$ & $-0,17$ & 0,02 & $-0,99$ \\
\hline Precio internacional del petróleo & 0,16 & 0,16 & 0,02 & 1,50 \\
\hline Precio internacional de palma & 0,06 & 0,13 & 0,02 & 0,43 \\
\hline Precio internacional del banano & $-0,11$ & 0,14 & 0,02 & $-0,97$ \\
\hline Precio internacional del azúcar & $-0,03$ & 0,15 & 0,02 & $-0,31$ \\
\hline Precio internacional del tabaco & $-0,15$ & 0,03 & 0,02 & $-0,27$ \\
\hline
\end{tabular}

Fuente: Cálculos propios a partir de la matriz de correlaciones. Cifras Banco Mundial y Federación Nacional de Cafeteros. 


\section{CONCLUSIONES}

Uno de los hechos macroeconómicos más importantes durante el periodo 1976 y 2013 fue el crecimiento persistente del PIB por trabajador del sector agropecuario, sobre todo en el periodo de 1980 a 2000. El crecimiento del PIB agropecuario durante este periodo se debe en gran parte al crecimiento del producto por trabajador y a la expansión en el cambio técnico medido a través de la PTF, resultado que está en consonancia con lo encontrado por autores como Kalmanovitz y López (2006).

La contribución del cambio tecnológico al crecimiento del PIB del sector ha presentado una tendencia creciente a lo largo de estos 37 años. Por su parte, la relación capital-producto ha presentado una tendencia decreciente, excepto en el periodo 1991 a 1995, en donde la participación del stock de capital del sector con relación al PIB se favoreció en parte por el incremento en la importación de tractores y maquinaria agrícola, en parte por el aumento en la tasa de ahorro. El análisis de contabilidad de crecimiento durante este periodo muestra una fuerte contribución del factor trabajo, baja contribución del stock de capital y fuerte contribución del crecimiento de la PTF.

Con relación a la estimación de los ciclos se registran tres ciclos completos para el sector agrícola con una duración de 16 trimestres (4 años) medidos de pico a pico, o de 15 trimestres (3,8 años) medidos de valle a valle. Las fases de expansión duran, en promedio, 29 trimestres $(9,6$ años), y las de contracción, 4 trimestres (un año), lo que implica que los ciclos en el sector agrícola son asimétricos, dada la mayor duración de las expansiones con relación a las contracciones.

Los ejercicios de impulso respuesta implican que las condiciones de la senda de crecimiento del PIB agropecuario colombiano se han modificado sustancialmente, en parte por las ganancias en productividad de los recursos productivos y en parte por el efecto de las políticas de oferta y demanda. En efecto, las fluctuaciones del PIB en el sector agropecuario han estado generadas por choques de oferta y de demanda simultáneamente. No obstante, los choques de demanda originados por booms de exportaciones de café y el buen comportamiento de los precios internacionales del café han tenido efectos favorables sobre el producto y sobre la consiguiente reducción en la tasa de desempleo del sector.

Las variables agregadas de la actividad agropecuaria se comportan de manera procíclica y se mueven en igual dirección que el PIB. La fuerte covarianza del PIB con el consumo aparente y de las exportaciones del café y la balanza comercial, es uno de los mejores hechos estilizados que han caracterizado las fluctuaciones económicas en el sector agrícola durante el periodo de análisis.

Finalmente, es necesario destacar del estudio que la importancia de una efectiva 
administración macroeconómica por parte de los "hacedores de política" va dirigida a propiciar el desarrollo de la economía y específicamente del sector agropecuario, mediante el diseño de políticas contracíclicas y estrategias que propendan a la diversificación productiva. De esta manera, la política anticíclica debe estar encaminada a enfrentar los retos que implican los agudos ciclos en el sector y las acentuadas fluctuaciones de los precios de productos básicos.

\section{REFERENCIAS}

Ahumada, H. \& Garegnani, M. L. (1999, abril). Hodrick-Prescott Filter in Practice. Buenos Aires: Universidad Nacional de La Plata.

Arango, L., Alfonso, V., Arias, F., Cangrejo, G. \& Pulido, J. (2013). Ciclos de negocios en Colombia, 1975-2011. Lecturas de Economía, (78).

Barro, R. \& Sala-i-Martin, X. (2004). Economic Growth. (Second ed.). Cambridge, Massachusetss: The MIT Press.

Berry, A. (1970). The Development of Colombian Agriculture. New Haven, USA: Yale University, Mimeo.

Blanchard, O. \& Fischer, S. (1986). Lectures on Macroeconomics. Cambridge, Massachusetss: The MIT Press.

Box, G.E.P. \& Jenkins, G. (1976). Time Series Analysis: Forecasting and Control. USA: Holden-Day.

Burns, A. \& Mitchell, W. (1946). Measuring Business Cycles. New York: National Bureau of Economic Research.

Campbell, J. \& Mankiw, N.G. (1987a). Are Output Fluctuations Transitory? Quarterly Journal of Economics 102(4).

Grupo de Estudios del Crecimiento Económico (GRECO), Banco de la República. (2004). El crecimiento económico colombiano en el siglo XX. Bogotá: Fondo de Cultura Económica. 
Hayashi, F. \& Prescott, E. (2002, February). The 1990s in Japan: a lost Decade. Rev. Econ. Dynamics, 5.

Hayashi, F. \& Prescott, E. (2008). The Depressing Effect of Agricultural Institutions on the Prewar Japanese Economy. Journal of Political Economy, 116(4).

Hodrick, R. J. \& Prescott, E.C. (1980). Postwar U.S. Business Cycles: an Empirical Investigation. Discussion Papers 451. Pittsburgh, USA: Carnegie-Mellon University, Northwestern University.

Jaramillo, C. F. (2002). Crisis y transformación de la agricultura colombiana 1990 2000. Bogotá: Fondo de Cultura Económica.

Kalmanovitz, S. \& López, E. (2006). La agricultura colombiana en el siglo XX. Bogotá: Fondo de Cultura Económica.

King, R. \& Rebelo, S. (1993). Low Frequency Filtering and Real Business Cycles. Journal of Economic Dynamics and Control, 17(1-2).

King, R. G. \& Ross, L. (1994, June). Capital Fundamentalism, Economic Development and Economic Growth. Carnegie -Rochester Conf. Ser. Public Policy, 40.

Klenow, P. J. \& Rodríguez-Clare, A. (1997). The Neoclassical Revival in Growth Economics: has it gone too far? In B. S. Bernanke \& J. Rotemberg (eds.) NBER Macroeconomics Annual. Cambridge, MA: MIT Press.

Londoño, J. L. (1984). Agricultura y transformación estructural. Una comparación internacional. Revista Planeación y Desarrollo, 21(3 y 4).

Nelson, C. \& Plosser, C. (1982, Sept.). Trends and Random Walks in Macroeconomic Time Series. Journal of Monetary Economics, 10.

Ocampo, J. A. (1984). Colombia y la economía mundial, 1830-1910. Bogotá: Siglo XXI.

Ocampo, J.A. (1991). The Transition from Primary Exports to Industrial Development in Colombia. In M. Blomstrom \& P. Mellev (eds.), Diverging Paths. Washington: s.n. 
Okun, A. M. (1962). Potential GNP: its Measurement and Significance. In J. Pechman (ed.) (1983), Economics for Policymaking, Cambridge, MA: MIT Press.

Prescott, E. (1986). Theory ahead of Business Cycle Measurement. Federal Reserve Bank of Minneapolis Quarterly Review 10(4).

Ravn, M. \& Uhlig, H. (1997). On adjusting the HP-Filter for the Frequency of Observations. Netherlands: Tilburg University Working Paper.

Solow, R. M. (1957, August). Technical Change and the Aggregate Production Function. The Review of Economics and Statistics, 39(3).

Uribe, A.F. (1998). Las grandes transformaciones del sector agropecuario colombiano en la última década: una visión regional. Bogotá: Corpoica.

Urrutia, M. \& Robinson, J. (2007). Economía colombiana del siglo XX. Un análisis cuantitativo. Bogotá: Fondo de Cultura Económica y Banco de la República.

Zarnowitz, V. (1992, January). Business Cycles: Theory, History, Indicators and Forecasting. Bogotá: University of Chicago Press. 


\section{ANEXO 1}

Pruebas de raíz unitaria

\begin{tabular}{|c|c|c|c|c|}
\hline \multicolumn{5}{|c|}{ Componente cíclico } \\
\hline \multirow{2}{*}{ Variable } & \multicolumn{2}{|c|}{ ADF } & \multicolumn{2}{|c|}{$\mathbf{P}-\mathbf{P}$} \\
\hline & t - estadístico ADF & Valores críticos & $\mathrm{Z}(\mathrm{t})$ - estadístico P-P & Valores críticos \\
\hline \multirow[t]{3}{*}{ PIB } & -3.648 & $(1 \%):-3.494$ & -5.634 & $(1 \%):-4.023$ \\
\hline & & $(5 \%):-2.887$ & & $(5 \%):-3.443$ \\
\hline & & $(10 \%):-2.577$ & & $(10 \%):-3.143$ \\
\hline \multirow[t]{3}{*}{ Empleo } & -4.201 & $(1 \%):-4.024$ & -8.739 & $(1 \%):-4.023$ \\
\hline & & $(5 \%):-3.444$ & & $(5 \%):-3.443$ \\
\hline & & $(10 \%):-3.144$ & & $(10 \%):-3.143$ \\
\hline \multicolumn{5}{|c|}{ Componente tendencia } \\
\hline \multirow{2}{*}{ Variable } & \multicolumn{2}{|c|}{ ADF } & \multicolumn{2}{|c|}{$\mathbf{P}-\mathbf{P}$} \\
\hline & t - estadístico ADF & Valores críticos & $Z(t)$ - estadístico P-P & Valores críticos \\
\hline \multirow[t]{3}{*}{ PIB } & -0.825 & $(1 \%):-4.024$ & -1.496 & $(1 \%):-4.023$ \\
\hline & & $(5 \%):-3.444$ & & $(5 \%):-3.443$ \\
\hline & & $(10 \%):-3.144$ & & $(10 \%):-3.143$ \\
\hline \multirow[t]{3}{*}{ Empleo } & -1.712 & $(1 \%):-3.494$ & -1.574 & $(1 \%):-4.023$ \\
\hline & & $(5 \%):-2.887$ & & $(5 \%):-3.443$ \\
\hline & & $(10 \%):-2.577$ & & $(10 \%):-3.143$ \\
\hline
\end{tabular}

Nota: la hipótesis nula para contrastar en las dos pruebas es que la serie es no estacionaria o por lo menos presenta una raíz unitaria. 


\section{ANEXO 2}

\begin{tabular}{|l|c|c|c|c|c|c|}
\hline \multicolumn{7}{|c|}{ Estadísticas descriptivas } \\
\hline \multicolumn{1}{|c|}{ Variable } & Máximo & Mínimo & Promedio & $\begin{array}{c}\text { Desv. } \\
\text { estándar }\end{array}$ & $\begin{array}{c}\text { Coeficiente } \\
\text { variación }\end{array}$ & N \\
\hline PIB & $28.995,0$ & $11.450,9$ & $20.160,7$ & $5.476,1$ & 0,27 & 37 \\
Empleo & $3.645,5$ & $1.505,8$ & $2.601,3$ & 676,1 & 0,26 & 37 \\
PIB por trabajador & 8,9 & 7,0 & 7,9 & 0,6 & 0,07 & 37 \\
Stock de capital & $38.590,4$ & $22.460,9$ & $32.217,9$ & $4.226,8$ & 0,13 & 37 \\
Horas trabajadas & $3.681,4$ & $1.677,6$ & $2.717,3$ & 641,9 & 0,24 & 37 \\
Consumo aparente & $7.124,2$ & $4.543,4$ & $4.543,4$ & $1.345,1$ & 0,30 & 37 \\
Exportaciones totales & $1.473,4$ & 258,8 & 718,4 & 270,6 & 0,38 & 37 \\
Exportaciones de café & 935,1 & 120,4 & 120,4 & 140,0 & 1,16 & 37 \\
Balanza comercial & $1.002,6$ & 215,7 & 565,3 & 164,9 & 0,29 & 37 \\
Precio internacional del café & 301,5 & 58,9 & 140,1 & 52,5 & 0,37 & 37 \\
Precio internacional del petróleo & 121,0 & 11,5 & 36,6 & 28,6 & 0,78 & 37 \\
Precio internacional de palma & $1.251,0$ & 208,3 & 545,9 & 222,0 & 0,41 & 37 \\
Precio internacional del banano & 1,1 & 0,2 & 0,5 & 0,2 & 0,39 & 37 \\
Precio internacional del azúcar & 0,8 & 0,1 & 0,3 & 0,1 & 0,51 & 37 \\
Precio internacional del tabaco & $4.885,7$ & $2.046,7$ & $3.034,3$ & 656,3 & 0,22 & 37 \\
& & & & & & \\
\hline
\end{tabular}




\section{ANEXO 3}

\begin{tabular}{|c|c|c|}
\hline \multicolumn{3}{|c|}{ Fuentes y unidades para las variables } \\
\hline Variable & Fuentes & Unidad \\
\hline PIB & $\begin{array}{l}\text { DANE - Sistema de Cuentas } \\
\text { Nacionales. Ejercicio de empalme } \\
\text { mediante retropolación. }\end{array}$ & $\begin{array}{l}\text { Millones de pesos constantes } \\
\text { de } 2005 .\end{array}$ \\
\hline Empleo & $\begin{array}{l}\text { Juan Luis Londoño se completó } \\
\text { con ECH-DANE varios años. }\end{array}$ & Número de personas empleadas. \\
\hline PIB por trabajador & $\begin{array}{l}\text { Calculos propios sobre cifras } \\
\text { DANE. }\end{array}$ & Pesos por trabajador \\
\hline Stock de capital & $\begin{array}{l}\text { Cálculos propios con base en } \\
\text { técnica de inventario perpetuo y } \\
\text { trabajos DNP (Diego Vásquez y } \\
\text { Juan R. Perilla). }\end{array}$ & $\begin{array}{l}\text { Millones de pesos constantes } \\
\text { de } 2005 .\end{array}$ \\
\hline Horas trabajadas & $\begin{array}{l}\text { Cálculos propios con base en } \\
\text { información del MADR. }\end{array}$ & Número de horas. \\
\hline Consumo aparente & $\begin{array}{l}\text { Calculos propios sobre cifras } \\
\text { DANE - DIAN }\end{array}$ & $\begin{array}{l}\text { Millones de pesos constantes } \\
\text { de } 2005 .\end{array}$ \\
\hline Exportaciones totales & DANE - DIAN & Milones de dólares. \\
\hline Exportaciones de café & DANE - DIAN & Milones de dólares. \\
\hline Balanza comercial & $\begin{array}{l}\text { Calculos propios sobre cifras } \\
\text { DANE-DIAN }\end{array}$ & Milones de dólares. \\
\hline Precio internacional del café & $\begin{array}{l}\text { Federación de Cafeteros de } \\
\text { Colombia. }\end{array}$ & $\begin{array}{l}\text { Centavos de dólar por libra de } \\
453.6 \text { gr. Excelso }\end{array}$ \\
\hline Precio internacional del petróleo & Bloomberg & Dólares por barril WTI \\
\hline Precio internacional de palma & Banco Mundial & Dólares nominales por kilogramo \\
\hline Precio internacional del banano & Banco Mundial & Dólares nominales por kilogramo \\
\hline Precio internacional del azúcar & Banco Mundial & Dólares nominales por kilogramo \\
\hline Precio internacional del tabaco & Banco Mundial & Dólares nominales por kilogramo \\
\hline
\end{tabular}

